\title{
Nevada
}

Environmental

Restoration

Project

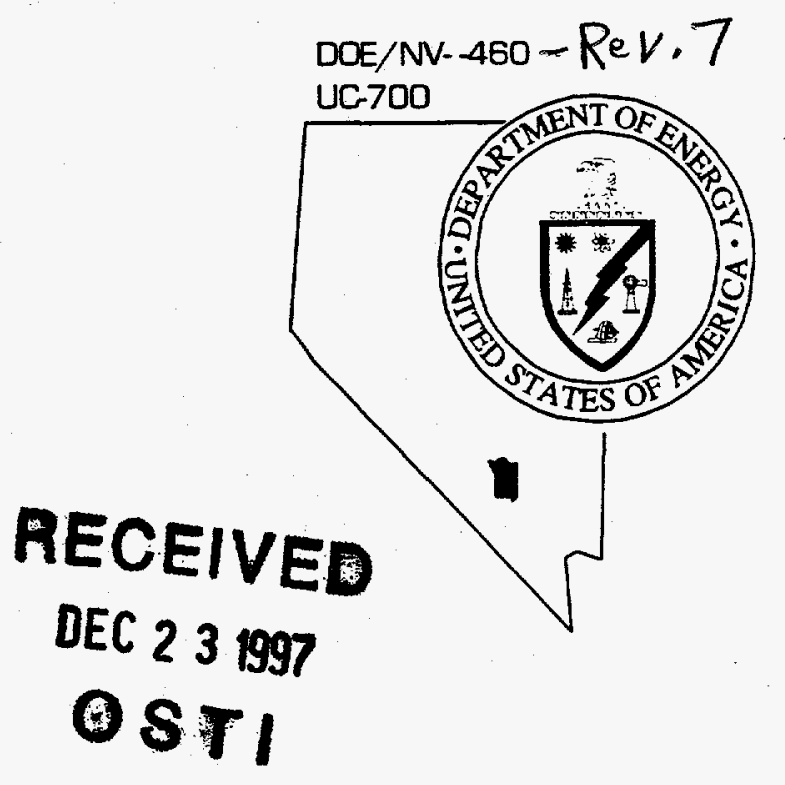

Rulison Site

Groundwater Monitoring Report

Third Quarter, 1997

October 1997

BSTRIBUTION OF THIS DOCUMENT IS UNUMITED

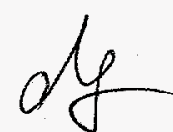

Approved for public release; further dissemination unlimited.

Environmental Restoration

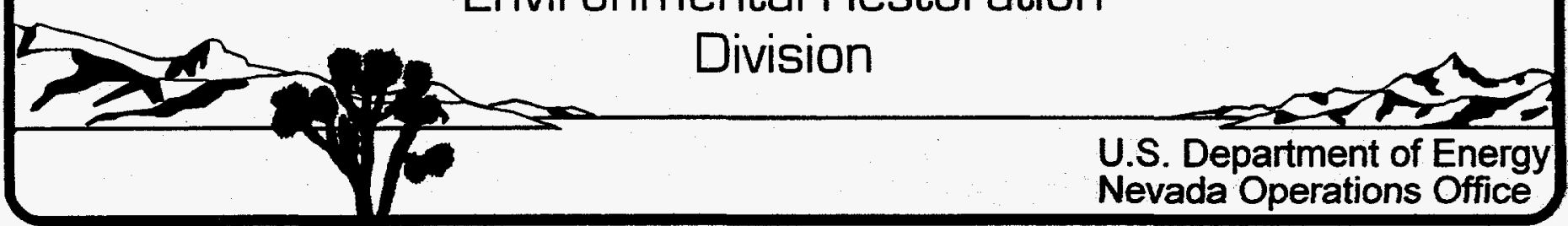

MASTER 
This report has been reproduced directly from the best available copy.

Available to DOE and DOE contractors from the Office of Scientific and Technical Information, P.O. Box 62, Oak Ridge, TN 37831; prices available from (423) 576-8401.

Available to the public from the National Technical Information Service, U.S. Department of Commerce, 5285 Port Royal Rd., Springfield, VA 22161, telephone (703) 487-4650. 


\section{RULISON SITE GROUNDWATER MONITORING REPORT THIRD QUARTER, 1997}

DOE Nevada Operations Office

Las Vegas, Nevada

October 1997

Approved for public release; further dissemination unlimited. 


\section{DISCLAIMER}

Portions of this document may be illegible electronic image products. Images are produced from the best available original document. 


\section{RULISON SITE \\ GROUNDWATER MONITORING REPORT \\ THIRD QUARTER, 1997}

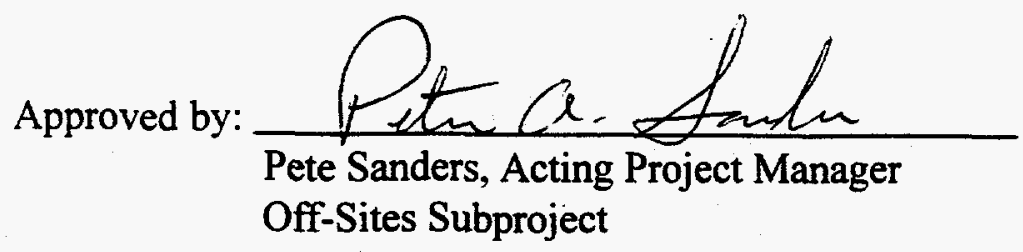

Date: $10 / 23 / 97$

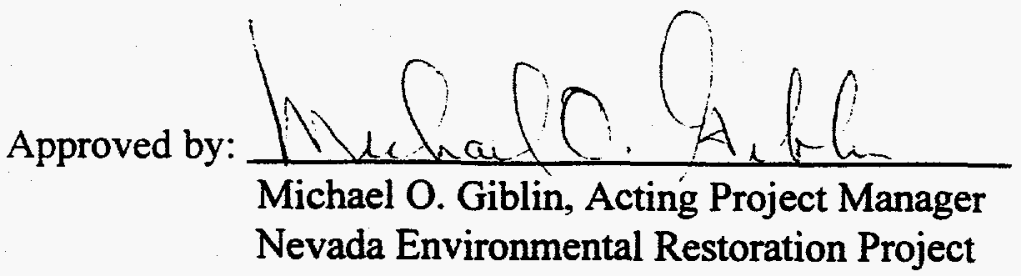

Date: $\frac{10 / 25 / 97}{1 / 27}$ 


\section{Table of Contents}

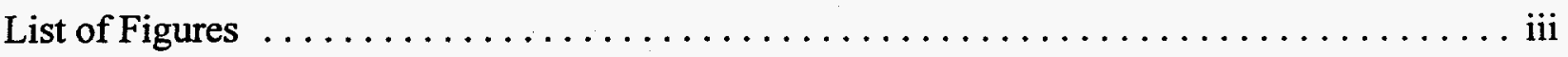

List of Tables $\ldots \ldots \ldots \ldots \ldots \ldots \ldots \ldots \ldots \ldots \ldots \ldots \ldots \ldots \ldots \ldots \ldots$ iii

List of Acronyms and Abbreviations $\ldots \ldots \ldots \ldots \ldots \ldots \ldots \ldots \ldots \ldots \ldots \ldots \ldots \ldots$

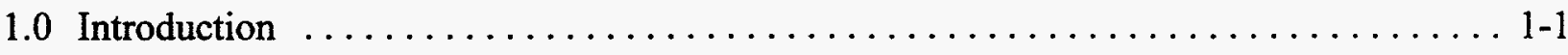

1.1 Site Location $\ldots \ldots \ldots \ldots \ldots \ldots \ldots \ldots \ldots \ldots \ldots \ldots \ldots \ldots \ldots \ldots \ldots \ldots \ldots \ldots \ldots \ldots \ldots \ldots$

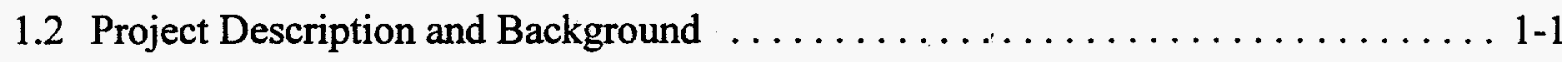

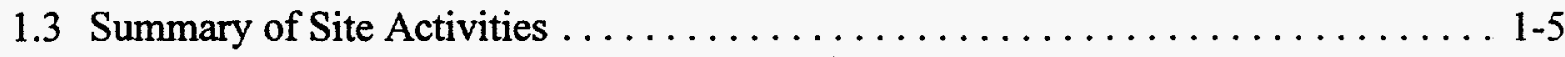

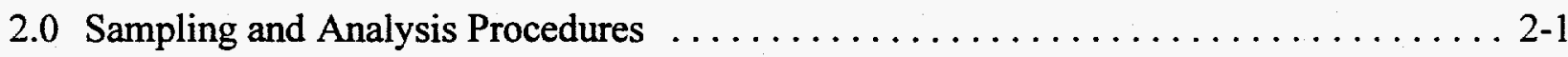

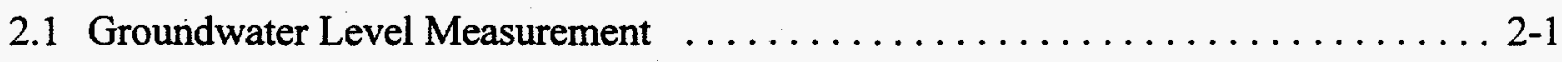

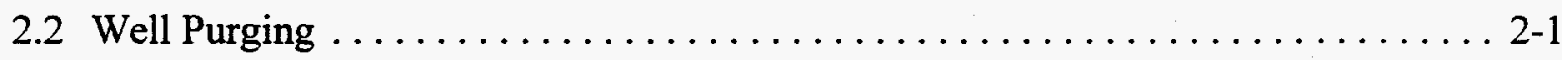

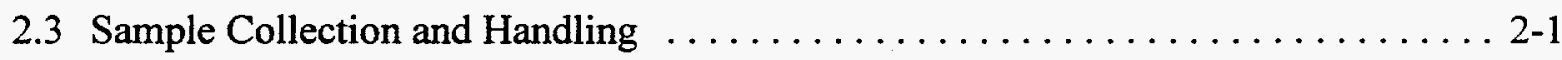

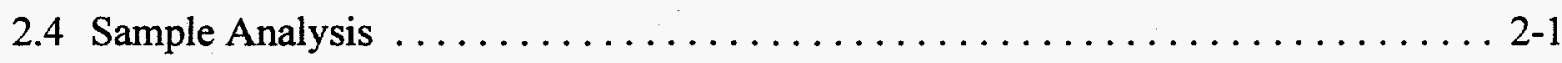

3.0 Analytical Results $\ldots \ldots \ldots \ldots \ldots \ldots \ldots \ldots \ldots \ldots \ldots \ldots \ldots \ldots \ldots \ldots \ldots \ldots$

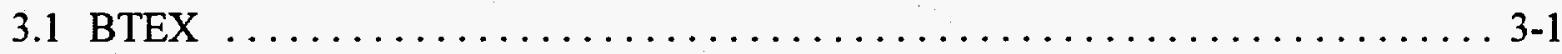

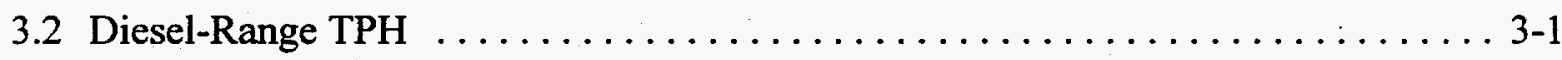

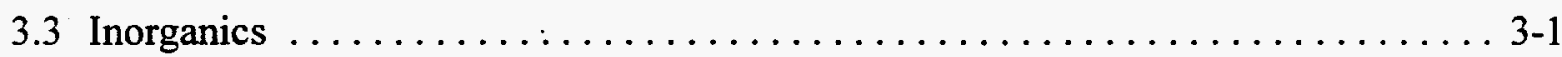

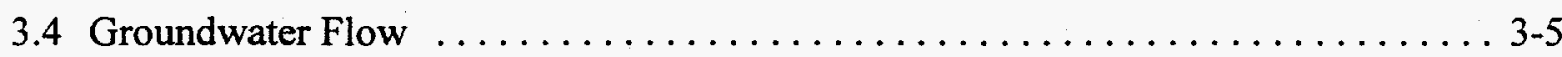

4.0 Quality Control Results $\ldots \ldots \ldots \ldots \ldots \ldots \ldots \ldots \ldots \ldots \ldots \ldots \ldots \ldots \ldots \ldots \ldots$

4.1 Field Duplicate Samples . . . . . . . . . . . . . . . .

4.2 Equipment Rinsate Blank Samples . . . . . . . . . . . . . . . .

4.3 Trip Blank Samples . . . . . . . . . . . . . . . . . . . . . 4-2

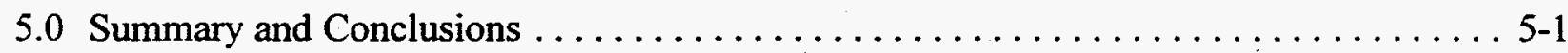

6.0 References $\ldots \ldots \ldots \ldots \ldots \ldots \ldots \ldots \ldots \ldots \ldots \ldots \ldots \ldots \ldots \ldots \ldots \ldots \ldots \ldots \ldots$ 


\section{Table of Contents (Continued)}

Appendix A - Purge Water Discharge Permit $\ldots \ldots \ldots \ldots \ldots \ldots \ldots \ldots \ldots \ldots \ldots \ldots$ A-1

Appendix B - Third Quarter 1997 Analytical Results . . . . . . . . . . . . . . . . B-1 


\section{List of Figures}

Number

Title

Page

1-1 Rulison Site Location Map

$1-2$

$1-2$

Monitoring Well Locations

$1-3$

\section{List of Tables}

Number

Title

Page

2-1 Rulison Site Groundwater Monitoring Program

Sample Container, Preservation, and Analytical Requirements

3-1 Rulison Site Groundwater Analytical Results

Third Quarter, 1997

3-2 Rulison Site Groundwater Elevations

Third Quarter, 1997

4-1 Rulison Site Groundwater Monitoring Program Duplicate Sample

Comparison: Third Quarter, 1997

B-1 Sample Number and Description 


\section{List of Acronyms and Abbreviations}

\begin{tabular}{|c|c|}
\hline AEC & U.S. Atomic Energy Commission \\
\hline Austral & Austral Oil Company \\
\hline BTEX & Benzene, toluene, ethylbenzene, and xylenes \\
\hline COPC & Constituent(s) of potential concern \\
\hline DOE & U.S. Department of Energy \\
\hline EPA & U.S. Environmental Protection Agency \\
\hline $\mathrm{ft}$ & Foot (feet) \\
\hline $\mathrm{H}_{2} \mathrm{SO}_{4}$ & Sulfuric acid \\
\hline $\mathrm{HCl}$ & Hydrochloric acid \\
\hline $\mathrm{HNO}_{3}$ & Nitric acid \\
\hline $\mathrm{km}$ & Kilometer(s) \\
\hline LTGMP & Long-Term Groundwater Monitoring Plan \\
\hline $\mathrm{m}$ & Meter(s) \\
\hline $\mathrm{mi}$ & Mile(s) \\
\hline $\mathrm{mL}$ & Milliliter(s) \\
\hline MS/MSD & Matrix spike/matrix spike duplicate \\
\hline QAPP & Quality Assurance Project Plan \\
\hline QC & Quality control \\
\hline RCRA & Resource Conservation and Recovery Act \\
\hline RPD & Relative percent difference(s) \\
\hline SGZ & Surface ground zero \\
\hline TPH & Total petroleum hydrocarbons \\
\hline TDS & Total dissolved solids \\
\hline TSS & Total suspended solids \\
\hline$\mu g / L$ & Microgram(s) per liter \\
\hline VOC & Volatile organic compound \\
\hline${ }^{\circ} \mathrm{F}$ & Degree(s) Fahrenheit \\
\hline
\end{tabular}




\subsection{Introduction}

This report summarizes the results of the third quarter 1997 groundwater sampling event for the Rulison Site, which is located approximately 65 kilometers $(\mathrm{km})$ (40 miles [mi]) northeast of Grand Junction, Colorado. The sampling was performed as part of a quarterly groundwater monitoring program implemented by the U.S. Department of Energy (DOE) to monitor the effectiveness of remediation of a drilling effluent pond located at the site. The effluent pond was used for the storage of drilling mud during drilling of the emplacement hole for a 1969 gas stimulation test conducted by the U.S. Atomic Energy Commission (AEC) (the predecessor agency to the DOE), and Austral Oil Company (Austral).

\subsection{Site Location}

The Rulison Site is located in the North $1 / 2$ of the Southwest $1 / 4$ of Section 25 , Township 7 South, Range 95 West of the $6^{\text {th }}$ Principal Meridian, Garfield County, Colorado, approximately $19 \mathrm{~km}$ (12 mi) southwest of Rifle, Colorado, and approximately $65 \mathrm{~km}$ (40 mi) northeast of Grand Junction, Colorado (Figure 1-1). The site is situated on the north slope of Battlement Mesa on the upper reaches of Battlement Creek, at an elevation of approximately 2,500 meters (m) $(8,200 \mathrm{feet}[\mathrm{ft}])$. The valley is open to the north-northwest and is bounded on the other three sides by steep mountain slopes that rise to elevations above $2,927 \mathrm{~m}(9,600 \mathrm{ft})$.

\subsection{Project Description and Background}

Project Rulison, a joint AEC and Austral experiment, was conducted under the AEC's Plowshare Program to evaluate the feasibility of using a nuclear device to stimulate natural gas production in low-permeability, gas-producing geologic formations. The experiment was conducted on September 10, 1969, and consisted of detonating a 40-kiloton nuclear device at a depth of $2,568 \mathrm{~m}(8,426 \mathrm{ft})$ below ground surface. Natural gas production testing was conducted in 1970 and 1971.

The site was deactivated by the AEC and Austral in 1972 and abandoned in 1976. Cleanup associated with site abandonment consisted of removing all remaining equipment and materials, plugging the emplacement (R-E) and reentry (R-EX) wells (Figure 1-2), backfilling the mud pits adjacent to the R-EX well, removing the tritium-contaminated soils, and conducting extensive surface soil sampling and analysis to characterize the radiological condition of the site. 


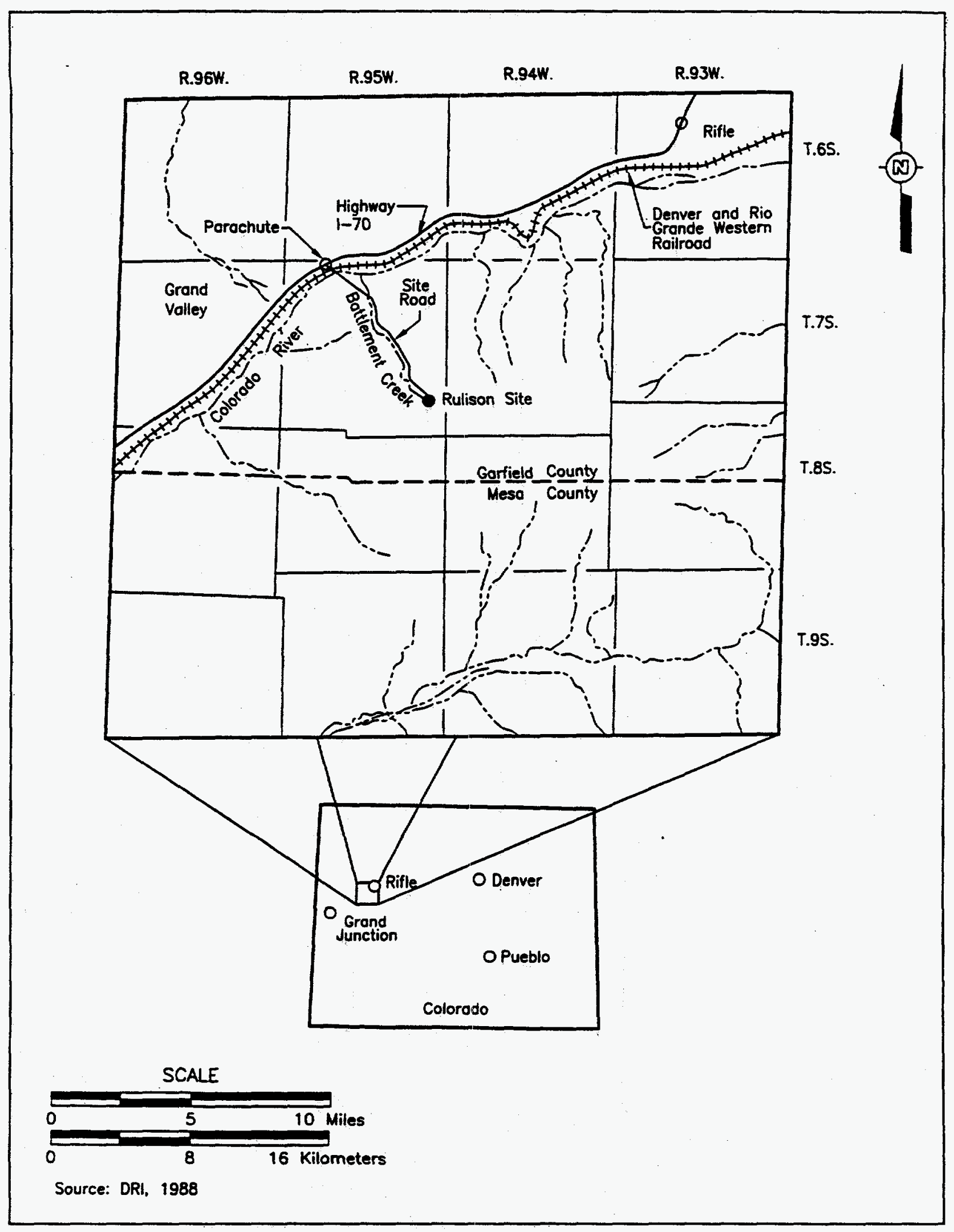

Figure 1-1

Rulison Site Location Map 


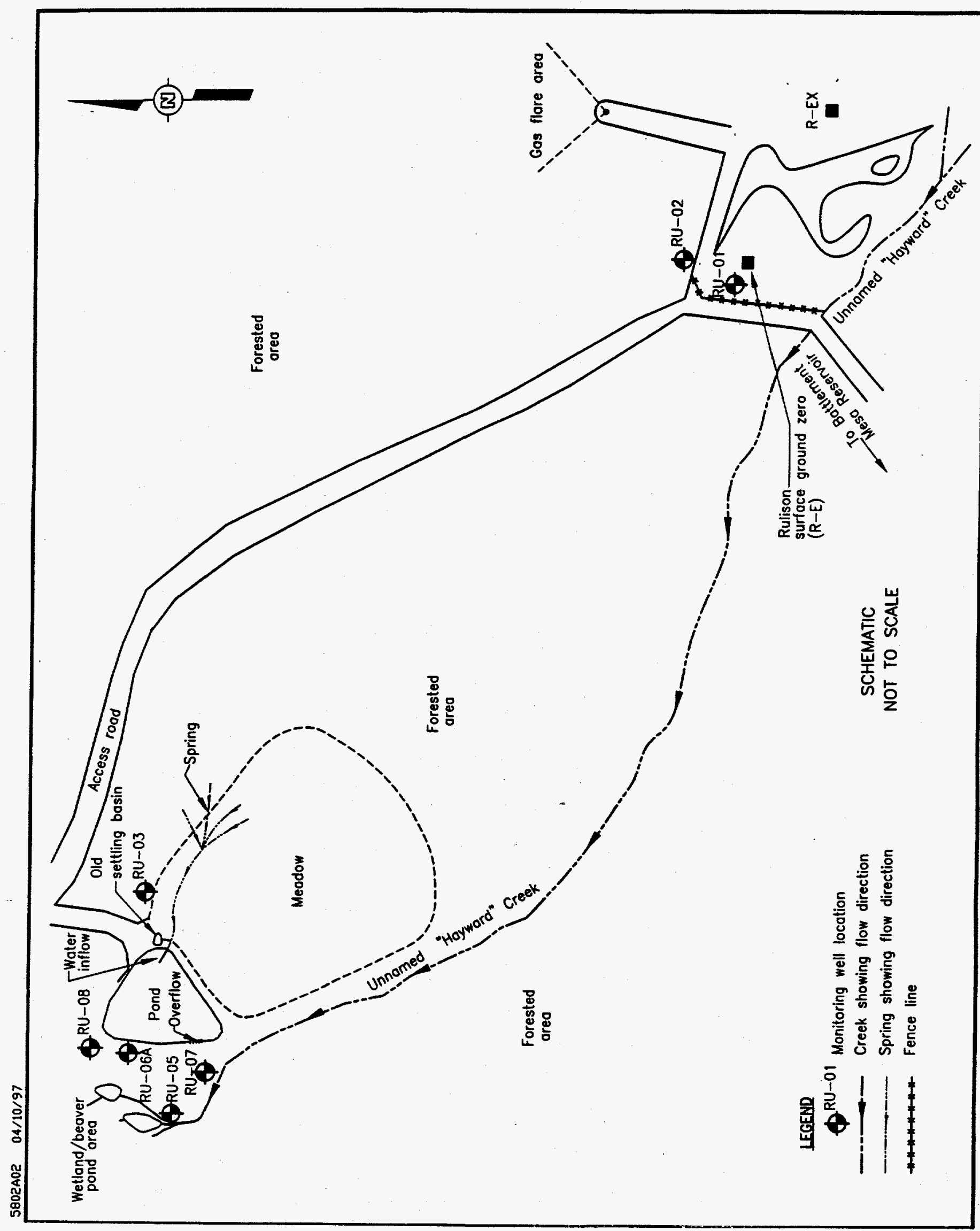

Figure 1-2

Monitoring Well Locations 
Detailed descriptions of the site deactivation and abandonment activities and radiological characterizations are presented in the Rulison Site Cleanup Report (AEC, 1973), the Project Rulison Well Plugging and Site Abandonment Final Report (ERDA, 1977), and the Rulison Radiation Contamination Clearance Report (Eberline, 1977).

The drilling effluent pond is an engineered structure located approximately $400 \mathrm{~m}(1,312 \mathrm{ft})$ north-northwest of the surface ground zero (SGZ) emplacement well (R-E) (Figure 1-2). The pond covers approximately 0.5 hectare (1.2 acres) as measured at the top of the berm; it is

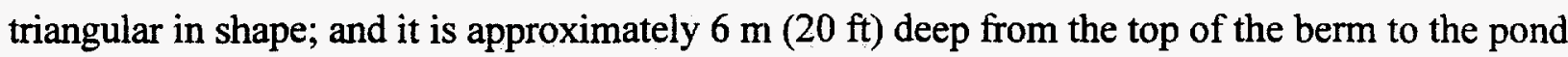
bottom. The drilling effluent pond was used to store nonradioactive drilling fluids generated during drilling of the device emplacement Well R-E. The drilling fluids consisted of bentonite drilling mud that contained various additives, such as diesel fuel and chrome lignosulfonate, used to improve drilling characteristics. Most of the drilling wastes were removed from the pond when the site was cleaned up and decommissioned in 1976; however, some drilling fluid was left in the pond. At the request of the property owner, the pond structure was left in place following completion of site decommissioning and was subsequently converted by the property owner to a freshwater holding pond containing aquatic vegetation, amphibians, and stocked rainbow trout.

In 1994 and 1995, four pond sediment sampling events were conducted to evaluate the extent of residual contamination from drilling wastes remaining in the pond. Concentrations of dieselrange total petroleum hydrocarbons (TPH); benzene, toluene, ethylbenzene, and total xylenes (BTEX compounds); barium; chromium; and lead were found in pond sediment samples and soil samples taken from an old settling basin located adjacent to the pond. Based on the results of the 1994 and 1995 sampling events, the DOE decided to conduct a voluntary cleanup action at the pond to reduce the levels of TPH and chromium in pond sediments and soils in and adjacent to the pond. The cleanup was completed in November 1995. One upgradient monitoring well (RU-03 on Figure 1-2) and four downgradient monitoring wells (RU-05, RU-06A, RU-07, and RU-08) were installed around the pond to monitor the effectiveness of the cleanup. A detailed description of pond cleanup and well installation is presented in the Rulison Site Corrective Action Report (DOE, 1996b). 


\subsection{Summary of Site Activities}

The third quarter 1997 sampling event was conducted on August 21, 1997, by personnel from IT Corporation representing the U.S. Department of Energy, Nevada Operations Office. Four out of the five wells scheduled for sampling had a sufficient volume of water to be sampled. The weather was partly cloudy and the temperatures were generally in the $70^{\circ}$ degrees

Fahrenheit $\left({ }^{\circ} \mathrm{F}\right)$ with light winds. There was a brief afternoon rain shower that occurred between sampling wells. No other unusual observations were made. 


\subsection{Sampling and Analysis Procedures}

The third quarter 1997 groundwater sampling event was conducted in general accordance with the Rulison Drilling Effluent Pond Site Long-Term Groundwater Monitoring Plan (LTGMP) (DOE, 1996a) and the Rulison Site Quality Assurance Project Plan, Rulison Site, Colorado (QAPP) (DOE, 1996c).

\subsection{Groundwater Level Measurement}

Before purging and sampling activities at each well began, the depth to groundwater and total depth of the well were measured. This information was used to calculate the appropriate purge volume and to allow evaluation of any potential changes to groundwater flow direction since the previous sampling event.

\subsection{Well Purging}

Monitoring wells were purged of stagnant groundwater using disposable bailers. The $\mathrm{pH}$, temperature, and conductivity of the groundwater were taken prior to discharging any water to the surface and at regular intervals thereafter. The $\mathrm{pH}$ values ranged from 6.86 to 7.42 . The purge water was discharged to the ground under Colorado Wastewater Discharge Permit No. COG-310084 as approved by the Colorado Department of Public Health and Environment, Water Quality Control Division (see Appendix A).

\subsection{Sample Collection and Handling}

Groundwater samples were collected from Wells RU-03, RU-05, RU-06A and RU-08 with disposable bottom-emptying bailers. For quality control (QC) purposes, one duplicate sample, one matrix spike/matrix spike duplicate (MS/MSD), and an equipment rinse blank sample were collected during the sampling event. In addition, a trip blank accompanied all volatile organic samples in their shipping container. Samples were containerized and preserved as specified in Table 2-1. All containers were certified clean by the laboratory and remained sealed until ready for use.

\subsection{Sample Analysis}

The groundwater samples from the third quarter 1997 sampling event were analyzed for the parameters listed in Table 2-1, as specified in the Rulison LTGMP (DOE, 1996a). These 
parameters included the constituents of potential concern (COPCs) identified for the drilling effluent pond sediments (TPH, BTEX, chromium, iron, zinc, and lead).

Table 2-1

Rulison Site Groundwater Monitoring Program Sample Container, Preservation, and Analytical Requirements

\begin{tabular}{|c|c|c|c|c|c|}
\hline Parameter & $\begin{array}{l}\text { Analytical } \\
\text { Method }\end{array}$ & $\begin{array}{l}\text { Sample } \\
\text { Container }\end{array}$ & $\begin{array}{l}\text { Minimum } \\
\text { Amount of } \\
\text { Sample } \\
\text { Required }\end{array}$ & Holding Time & Preservative $^{\mathrm{a}}$ \\
\hline BTEX & $S W-846^{b} 8020 A$ & $\begin{array}{c}\text { Glass with } \\
\text { Teflon }{ }^{\mathrm{TM}} \text { - lined } \\
\text { cap }\end{array}$ & $3 \times 40 \mathrm{~mL}$ & 14 days & $\begin{array}{c}\mathrm{pH}<2 \text { with } \mathrm{HCl} \\
\text { Cool to } 4^{\circ} \mathrm{C}\end{array}$ \\
\hline $\begin{array}{c}\text { TPH } \\
\text { (diesel fraction) }\end{array}$ & SW-846 8015M ${ }^{C}$ & Amber Glas:s & 1 liter & 14 days & $\begin{array}{c}\mathrm{pH}<2 \text { with } \mathrm{H}_{2} \mathrm{SO}_{4} \\
\text { Cool to } 4^{\circ} \mathrm{C}\end{array}$ \\
\hline RCRA ${ }^{d}$ Metals & $\begin{array}{c}\text { SW-846 6010/ } \\
7470 A\end{array}$ & Polyethylene & 1 liter & 180 days & $\begin{array}{l}\mathrm{HNO}_{3} \text { to } \mathrm{pH}<2 \\
\text { Cool to } 4^{\circ} \mathrm{C} \text {. } \\
\text { unfittered }\end{array}$ \\
\hline $\begin{array}{c}\text { Total Dissolved Solids } \\
\text { (TDS) }\end{array}$ & EPA $160.1^{e}$ & Polyethylene & $100 \mathrm{~mL}$ & 7 days & Cool to $4^{\circ} \mathrm{C}$ \\
\hline $\begin{array}{l}\text { Total Suspended } \\
\text { Solids (TSS) }\end{array}$ & EPA $160.2^{e}$ & Polyethylene & $100 \mathrm{~mL}$ & 7 days & Cool to $4^{\circ} \mathrm{C}$ \\
\hline $\begin{array}{c}\text { Total Recoverable } \\
\text { Chromium, Iron and } \\
\text { Zinc }\end{array}$ & $\begin{array}{c}\text { SW-846 } \\
3005 / 6010 A\end{array}$ & Polyethylene: & 1 liter & 180 days & $\begin{array}{c}\text { pH }<2 \text { with } \mathrm{HNO}_{3} \\
\text { Cool to } 4^{\circ} \mathrm{C}\end{array}$ \\
\hline $\begin{array}{c}\text { Potentially Dissolved } \\
\text { Lead }\end{array}$ & SW-846 6010A & Polyethylens & 1 liter & 180 days & $\begin{array}{c}\mathrm{pH}<2 \text { with } \mathrm{HNO}_{3} \\
\text { Cool to } 4^{\circ} \mathrm{C}\end{array}$ \\
\hline
\end{tabular}

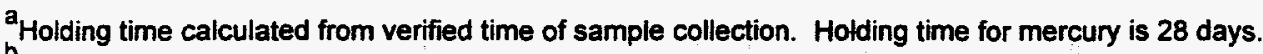

b.S. Environmental Protection Agency, SW-846, Test Methods for Evaluating Solid Waste, PhysicavChemical Methods, 3rd Edition (EPA, 1990)

CEPA SW-846, modified according to the California State Water Resources Control Board, Leaking Underground Fuel Tank Field Manual, Guidelines for Site Assessment, Cleanup, and Underground Storage Tank Closure, Appendix B (1989)

Resource Conservation and Recovery Act

U.S. Environmental Protection Agency, Methods for Chemical Analysis of Water and Wastes, (EPA, 1983)
$\mathrm{mL} \quad=$ Miltiliter(s)
$\mathrm{HCl}=$ Hydrochloric acid
$\mathrm{H}_{2} \mathrm{SO}_{4}=$ Sulfuric acid
$\mathrm{HNO}_{3}=$ Nitric acid
${ }^{\circ} \mathrm{C}=$ Degree(s) Celsius 


\subsection{Analytical Results}

The third quarter 1997 analytical results for the pond cleanup COPCs (diesel-range TPH, BTEX, barium, chromium, and lead) for the drilling effluent pond monitoring wells are presented in Table 3-1. Appendix B contains the laboratory report of the results for all analytes for the third quarter of 1997 sampling event. A review of the analytical data for laboratory method blanks was performed to ensure that the COPC concentrations reported for the groundwater samples were representative of groundwater quality rather than laboratory contamination. The following sections provide a discussion of the third quarter 1997 groundwater sampling results.

\subsection{BTEX}

Benzene and toluene were detected in Well RU-03, but not in any of the other wells. This is the first time these constituents have been detected in a well at the Rulison Site. The source is unknown. The presence of benzene and toluene in Well RU-03 is unrelated to the pond as RU-03 is hydrologically upgradient from the pond. Ethylbenzene and xylene were not detected in any of the groundwater samples from the third quarter 1997 sampling event. There were no data qualifiers for any of the samples.

\subsection{Diesel-Range TPH}

Diesel-range TPH was not detected in any of the groundwater samples from the third quarter 1997 sampling event.

\subsection{Inorganics}

The third quarter 1997 samples from all wells contained barium at levels ranging from 114 to 425 micrograms per liter $(\mu \mathrm{g} / \mathrm{L})$. Chromium was detected in two samples, upgradient Well RU-03 $(9.8 \mu \mathrm{g} / \mathrm{L})$ and downgradient Well RU-05 $(39.2 \mu \mathrm{g} / \mathrm{L})$. Chromium was not detected in Wells RU-06A and RU-08. The source of chromium in the groundwater is unknown, but it is suspected that it is naturally occurring in the soils at the Rulison Site. Its presence is not likely to represent migration from the pond sediments. During the second quarter 1997 sampling, arsenic was detected in all of the wells sampled (DOE, 1997); however, during the third quarter 1997 sampling, arsenic was detected in only two of the samples (RU-03 and RU-05). Arsenic was not identified as a COPC for pond cleanup and is likely to be of local natural origin. Lead was detected in three wells, RU-03, RU-05, and RU-08, and also in the equipment rinsate. Lead was not detected in Well RU-06A. Selenium was not detected in any of the samples. 
Table 3-1

Rulison Site Groundwater Analytical Results Third Quarter, 1997 (All results in $\mu \mathrm{g} / \mathrm{L}$ )

(Page 1 of 3 )

\begin{tabular}{|c|c|c|c|c|c|c|c|c|}
\hline Well & $\begin{array}{c}\text { First Quarter } \\
1996 \\
\end{array}$ & $\begin{array}{c}\text { Second Quarter } \\
1996 \\
\end{array}$ & $\begin{array}{c}\text { Third Quarter } \\
1996\end{array}$ & $\begin{array}{c}\text { Fourth Quarter } \\
1996\end{array}$ & $\begin{array}{c}\text { First Quarter } \\
1997 \\
\end{array}$ & $\begin{array}{c}\text { Second Quarter } \\
1997 \\
\end{array}$ & $\begin{array}{c}\text { Third Quarter } \\
1997\end{array}$ & $\begin{array}{c}\text { Fourth Quarter } \\
1997\end{array}$ \\
\hline \multicolumn{9}{|c|}{ TPH - Diesel } \\
\hline RU-03 & 1000 & $94 \mathrm{U}$ & 5000 & $500 \mathrm{U}$ & . 10000 & $1000 \mathrm{U}$ & $1000 \mathrm{U}$ & \\
\hline RU-05 & $100 \mathrm{UJ}^{1}$ & $94 \mathrm{U}$ & NS & NS & NS & 11000 & 10000 & \\
\hline RU-06A & 1000 & 71R & $500 \mathrm{U}$ & $500 U$ & $1000 \mathrm{U}$ & $1000 U$ & $1000 U$ & \\
\hline RU-07 & NS & NS & NS & NS & NS & NS & NS & \\
\hline RU-08 & $100 \mathrm{UJ}$ & $94 \mathrm{U}$ & NS & NS & NS & 13000 & $1000 U$ & \\
\hline & \multicolumn{8}{|c|}{ Benzene } \\
\hline RU.03 & $0.5 \mathrm{U}$ & $0.5 \mathrm{U}$ & 10 & 10 & 10 & $0.50 \mathrm{U}$ & 2.5 & \\
\hline RU-05 & $0.5 \mathrm{U}$ & $0.5 \mathrm{U}$ & NS & NS & NS & $0.50 U$ & $1.0 \mathrm{U}$ & \\
\hline RU-06A & 0.50 & $0.5 \mathrm{U}$ & 14 & 14 & 10 & $0.50 \mathrm{U}$ & 1.00 & \\
\hline RU.07 & NS & NS & NS & NS & NS & NS & NS & \\
\hline RU-08 & $0.5 \mathrm{U}$ & $0.5 \mathrm{U}$ & NS & NS & NS & $0.50 \mathrm{U}$ & 1.04 & \\
\hline & \multicolumn{8}{|c|}{ Toluene } \\
\hline RU-03 & $0.5 \mathrm{U}$ & $0.5 \mathrm{U}$ & 10 & $1 U$ & 10 & 1.00 & 3.9 & \\
\hline RU-05 & $0.5 \mathrm{U}$ & $0.5 \mathrm{U}$ & NS & NS & NS & $1.0 \mathrm{U}$ & 1.00 & \\
\hline RU-06A & $0.5 \mathrm{U}$ & $0.5 \mathrm{U}$ & 10 & $1 \mathrm{U}$ & 10 & 1.00 & $1.0 \mathrm{U}$ & \\
\hline RU-07 & NS & NS & NS & NS & NS & NS & NS & \\
\hline RU-08 & $0.5 \mathrm{U}$ & $0.5 \mathrm{U}$ & NS & NS & NS & 1.00 & $1.0 \mathrm{U}$ & \\
\hline \multicolumn{9}{|c|}{ Ethylbenzene } \\
\hline RU-03 & $0.5 \mathrm{U}$ & $0.5 \mathrm{U}$ & 10 & 10 & 10 & 1.00 & 1.00 & \\
\hline RU-05 & $0.5 \mathrm{U}$ & $0.5 \mathrm{U}$ & NS & NS & NS & 1.00 & $1.0 U$ & \\
\hline RU-06A & $0.5 \mathrm{U}$ & $0.5 \mathrm{U}$ & $1 U$ & 10 & 14 & 1.00 & 1.00 & \\
\hline RU-07 & NS & NS & NS & NS & NS & NS & NS & \\
\hline RU-08 & $0.5 \mathrm{U}$ & $0.5 \mathrm{U}$ & NS & NS & NS & $1.0 \mathrm{U}$ & 1.00 & \\
\hline
\end{tabular}


Table 3-1

Rulison Site Groundwater Analytical Results Third Quarter, 1997 (All results in $\mu \mathrm{g} / \mathrm{L}$ )

(Page 2 of 3)

\begin{tabular}{|c|c|c|c|c|c|c|c|c|}
\hline Well & $\begin{array}{c}\text { First Quarter } \\
1996 \\
\end{array}$ & $\begin{array}{c}\text { Second Quarter } \\
1996 \\
\end{array}$ & $\begin{array}{c}\text { Third Quarter } \\
1996\end{array}$ & $\begin{array}{c}\text { Fourth Quarter } \\
1996 \\
\end{array}$ & $\begin{array}{c}\text { First Quarter } \\
1997\end{array}$ & $\begin{array}{c}\text { Second Quarter } \\
1997 \\
\end{array}$ & $\begin{array}{c}\text { Third Quarter } \\
1997\end{array}$ & $\begin{array}{c}\text { Fourth Quarter } \\
1997 \\
\end{array}$ \\
\hline & \multicolumn{8}{|c|}{ Xylenes (total) } \\
\hline RU-03 & $0.5 U$ & $0.5 \mathrm{U}$ & 10 & 10 & 10 & $1.0 \mathrm{U}$ & 2.00 & \\
\hline RU.05 & $0.5 \mathrm{U}$ & $0.5 U$ & NS & NS & NS & 1.00 & 2.00 & \\
\hline RU-06A & $0.5 U$ & $0.5 \mathrm{U}$ & 10 & 10 & 10 & $1.0 \mathrm{U}$ & $2.0 \mathrm{U}$ & \\
\hline RU-07 & NS & NS & NS & NS & NS & NS & NS & \\
\hline RU-08 & $0.5 \mathrm{U}$ & $0.5 \mathrm{U}$ & NS & NS & NS & $1.0 \mathrm{U}$ & 2.00 & \\
\hline \multicolumn{9}{|c|}{ Barium } \\
\hline RU.03 & 120 & 110 & 105 & 135 & 86 & 90.3 & 148.0 & \\
\hline RU-05 & 360 & 120 & NS & NS & NS & 89.8 & 425.0 & \\
\hline RU-06A & 120 & 120 & 119 & 116 & 118 & 130 & 114.0 & \\
\hline RU-07 & NS & NS & NS & NS & NS & NS & NS & \\
\hline RU-08 & 350 & 140 & NS & NS & NS & 146 & 127.0 & \\
\hline & \multicolumn{8}{|c|}{ Chromium } \\
\hline RU-03 & $10 U$ & $10 U$ & $1.5 \mathrm{U}$ & 6.7 & 2.2 & 5.0 & 9.8 & \\
\hline RU-05 & 24 & 100 & NS & NS & NS & 1.8 & 39.2 & \\
\hline RU-06A & 100 & 100 & $1.5 \mathrm{U}$ & $\overline{1.5 \mathrm{U}}$ & 2.5 & $1.0 \mathrm{U}$ & 1.00 & \\
\hline RU-07 & NS & NS & NS & NS & NS & NS & NS & \\
\hline RU-08 & $10 \mathrm{U}$ & 100 & NS & NS & NS & 3.1 & $1.0 \mathrm{U}$ & \\
\hline & \multicolumn{8}{|c|}{ Lead } \\
\hline RU-03 & $5.6 \mathrm{U}$ & $3 U$ & 1.5 & $2.3 \mathrm{U}$ & 2.00 & 2.5 & 6.4 & \\
\hline RU-05 & $13 U$ & $3 U$ & NS & NS & NS & 3.1 & 18.5 & \\
\hline RU-06A & 34 & 30 & $0.8 U$ & $0.8 \mathrm{U}$ & 2.00 & 2.00 & $2.0 U$ & \\
\hline RU-07 & NS & NS & NS & NS & NS & NS & NS & \\
\hline $\mathrm{RU}-08$ & $12 U$ & 30 & NS & NS & NS & 3.5 & 2.5 & \\
\hline
\end{tabular}


Table 3-1

Rulison Site Groundwater Analytical Results

Third Quarter, 1997 (All results in $\mu \mathrm{g} / \mathrm{L}$ )

(Page 3 of 3)

\begin{tabular}{|c|c|c|c|c|c|c|c|c|}
\hline Well & $\begin{array}{c}\text { First Quarter } \\
1996 \\
\end{array}$ & $\begin{array}{c}\text { Second Quarter } \\
1996 \\
\end{array}$ & $\begin{array}{c}\text { Third Quarter } \\
1996 \\
\end{array}$ & $\begin{array}{c}\text { Fourth Quarter } \\
1996 \\
\end{array}$ & $\begin{array}{c}\text { First Quarter } \\
1997 \\
\end{array}$ & $\begin{array}{c}\text { Second Quarter } \\
1997 \\
\end{array}$ & $\begin{array}{c}\text { Third Quarter } \\
1997 \\
\end{array}$ & $\begin{array}{c}\text { Fourth Quarter } \\
1997 \\
\end{array}$ \\
\hline & \multicolumn{8}{|c|}{ Selenium } \\
\hline RU-03 & 16 & 14 & $2.8 \mathrm{U}$ & $2.8 \mathrm{U}$ & $4.0 \mathrm{U}$ & $3.0 \mathrm{U}$ & $3.0 \mathrm{U}$ & \\
\hline RU-05 & 7.2 & 6 & NS & NS & NS & $3.0 \mathrm{U}$ & $3.0 \mathrm{U}$ & \\
\hline RU-06A & 12 & 20 & $2.8 \mathrm{U}$ & $2.8 \mathrm{U}$ & $4.0 \mathrm{U}$ & $3.0 \mathrm{U}$ & $3.0 \mathrm{U}$ & \\
\hline RU-07 & NS & NS & NS & NS & NS & NS & NS & \\
\hline RU-08 & 12 & 22 & NS & NS & NS & $3.0 \mathrm{U}$ & $3.0 \mathrm{U}$ & \\
\hline
\end{tabular}

Values in italics are for the dissolved fraction

Values in bold are the third quarter 1997 sampling event results

NS = Well dry - no sample collected

$\stackrel{\omega}{b}$

$\mathrm{U}$ = Anaiyte not detected above the specified value

$R$ = Quality control indicates that the data are unusable (compound may or may not be present)

$\mathrm{J}=$ Reported value is estimated 
The relatively high concentrations of barium, chromium, and lead in Well RU-05 are most likely the result of sediment in the water sample. During previous sampling events, Well RU-05 often did not have enough water to sample. During the third quarter 1997 sampling event, Well RU-05 had 1 foot of water in it, the minimum amount of water required in order to sample. When this well was purged it was likely that sediment was stirred up from the bottom of the well and then picked up in the water sample.

There currently are insufficient data to establish concentration trends or to determine whether total barium concentrations in groundwater downgradient from the pond are significantly elevated above background level. Statistical trends will be calculated as data are acquired from additional quarterly groundwater monitoring events.

\subsection{Groundwater Flow}

Groundwater depth and elevation data for the drilling effluent pond monitoring wells from the third quarter 1997 sampling event are presented in Table 3-2. Based on the groundwater elevation data, it appears that groundwater flow during the third quarter sampling event was generally towards the northwest. Under this flow condition, Well RU-03 is upgradient from the pond, and Wells RU-05, RU-06A and RU-08 are downgradient from the pond. Well RU-05 is also upgradient of Wells RU-06A and RU-08. 
Table 3-2

Rulison Site Groundwater Elevations

Third Quarter, 1997

\begin{tabular}{|c|c|c|c|c|c|c|c|c|}
\hline Well & $\begin{array}{c}\text { First Quarter } \\
1996 \\
\end{array}$ & $\begin{array}{c}\text { Second Quarter } \\
1996 \\
\end{array}$ & $\begin{array}{c}\text { Third Quarter } \\
1996 \\
\end{array}$ & $\begin{array}{c}\text { Fourth Quarter } \\
1996 \\
\end{array}$ & $\begin{array}{c}\text { First Quarter } \\
1997 \\
\end{array}$ & $\begin{array}{c}\text { Second Quarter } \\
1997 \\
\end{array}$ & $\begin{array}{c}\text { Third Quarter } \\
1997 \\
\end{array}$ & $\begin{array}{c}\text { Fourth Quarter } \\
1997 \\
\end{array}$ \\
\hline \multicolumn{9}{|c|}{ Depth to Water (from top of casing) } \\
\hline RU-03 & $\begin{array}{r}10.56 \mathrm{~m} \\
(34.65 \mathrm{ft})\end{array}$ & $\begin{array}{c}6.81 \mathrm{~m} \\
(22.33 \mathrm{ft}) \\
\end{array}$ & $\begin{array}{l}12.94 \mathrm{~m} \\
(42.44 \mathrm{ft})\end{array}$ & $\begin{array}{r}12.93 \mathrm{~m} \\
(42.42 \mathrm{ft}) \\
\end{array}$ & $\begin{array}{r}10.90 \mathrm{~m} \\
(35.75 \mathrm{ft}) \\
\end{array}$ & $\begin{array}{r}3.82 \mathrm{~m} \\
(12.52 \mathrm{ft}) \\
\end{array}$ & $\begin{array}{l}8.68 \mathrm{~m} \\
(28.48 \mathrm{ft})\end{array}$ & \\
\hline RU-05 & $\begin{array}{l}2.35 \mathrm{~m} \\
(7.71 \mathrm{ft}) \\
\end{array}$ & $\begin{array}{r}1.96 \mathrm{~m} \\
(6.42 \mathrm{ft}) \\
\end{array}$ & Dry & Dry & $\begin{array}{l}3.05 \mathrm{~m}^{\mathrm{a}} \\
(10.0 \mathrm{ft})\end{array}$ & $\begin{array}{r}1.75 \mathrm{~m} \\
(5.75 \mathrm{ft}) \\
\end{array}$ & $\begin{array}{c}2.79 \mathrm{~m} \\
(9.15 \mathrm{ft}) \\
\end{array}$ & \\
\hline RU-06A & $\begin{array}{c}4.74 \mathrm{~m} \\
(15.56 \mathrm{ft})\end{array}$ & $\begin{array}{c}4.38 \mathrm{~m} \\
(14.38 \mathrm{ft}) \\
\end{array}$ & $\begin{array}{c}5.55 \mathrm{~m} \\
(18.20 \mathrm{ft}) \\
\end{array}$ & $\begin{array}{r}4.72 \mathrm{~m} \\
(15.5 \mathrm{ft}) \\
\end{array}$ & $\begin{array}{c}5.66 \mathrm{~m} \\
(18.56 \mathrm{ft}) \\
\end{array}$ & $\begin{array}{r}3.79 \mathrm{~m} \\
(12.45 \mathrm{ft}) \\
\end{array}$ & $\begin{array}{c}4.67 \mathrm{~m} \\
(15.32 \mathrm{ft})\end{array}$ & \\
\hline RU-07 & Dry & Dry & Dry & Dry & $\begin{array}{l}3.11 \mathrm{~m}^{\mathrm{a}} \\
(10.2 \mathrm{ft})\end{array}$ & Dry & Dry & \\
\hline RU-08 & $\begin{array}{r}1.78 \mathrm{~m} \\
(5.85 \mathrm{ft}) \\
\end{array}$ & $\begin{array}{r}1.70 \mathrm{~m} \\
(5.58 \mathrm{ft}) \\
\end{array}$ & Dry & Dry & $\begin{array}{c}2.23 \mathrm{~m}^{\mathrm{a}} \\
(7.3 \mathrm{ft}) \\
\end{array}$ & $\begin{array}{r}1.49 \mathrm{~m} \\
(4.9 \mathrm{ft}) \\
\end{array}$ & $\begin{array}{r}1.84 \mathrm{~m} \\
(6.04 \mathrm{tt}) \\
\end{array}$ & \\
\hline \multicolumn{9}{|c|}{ Groundwater Elevation } \\
\hline RU $=03$ & $\begin{array}{l}2444.29 \mathrm{~m} \\
(8019.33 \mathrm{ft}) \\
\end{array}$ & $\begin{array}{r}2446.05 \mathrm{~m} \\
(8031.65 \mathrm{ft}) \\
\end{array}$ & $\begin{array}{l}2441.92 \mathrm{~m} \\
(8011.54 \mathrm{ft}) \\
\end{array}$ & $\begin{array}{r}2441.92 \mathrm{~m} \\
(8011.56 \mathrm{ft}) \\
\end{array}$ & $\begin{array}{r}2443.96 \mathrm{~m} \\
(8018.23 \mathrm{ft})\end{array}$ & $\begin{array}{r}2451.04 \mathrm{~m} \\
(8041.46 \mathrm{ft}) \\
\end{array}$ & $\begin{array}{l}2446.17 \mathrm{~m} \\
(8025.5 \mathrm{ft})\end{array}$ & \\
\hline RU-05 & $\begin{array}{r}2433.95 \mathrm{~m} \\
(7985.41 \mathrm{ft}) \\
\end{array}$ & $\begin{array}{l}2434.35 \mathrm{~m} \\
(7986.70 \mathrm{ft})\end{array}$ & $\begin{array}{l}<2434.09 \mathrm{~m} \\
(<7985.87 \mathrm{ft}) \\
\end{array}$ & $\begin{array}{l}<2434.09 \mathrm{~m} \\
(<7985.87 \mathrm{ft})\end{array}$ & $\begin{array}{l}2433.26 \mathrm{~m} \\
(7983.12 \mathrm{ft}) \\
\end{array}$ & $\begin{array}{l}2434.55 \mathrm{~m} \\
(7987.37 \mathrm{ft}) \\
\end{array}$ & $\begin{array}{l}2433.51 \mathrm{~m} \\
(7983.97 \mathrm{ft}) \\
\end{array}$ & \\
\hline RU-06A & $\begin{array}{r}2430.10 \mathrm{~m} \\
(7972.78 \mathrm{ft}) \\
\end{array}$ & $\begin{array}{r}2430.46 \mathrm{~m} \\
(7973.96 \mathrm{ft}) \\
\end{array}$ & $\begin{array}{r}2429.30 \mathrm{~m} \\
(7970.14 \mathrm{ft}) \\
\end{array}$ & $\begin{array}{r}2430.12 \mathrm{~m} \\
(7972.84 \mathrm{ft}) \\
\end{array}$ & $\begin{array}{r}2429.19 \mathrm{~m} \\
(7969.78 \mathrm{ft}) \\
\end{array}$ & $\begin{array}{r}2431.05 \mathrm{~m} \\
(7975.89 \mathrm{ft}) \\
\end{array}$ & $\begin{array}{c}2430.18 \mathrm{~m} \\
(7973.02 \mathrm{ft})\end{array}$ & \\
\hline RU-07 & $\begin{array}{r}<2438.22 \mathrm{~m}_{\mathrm{b}} \\
(<7999.40 \mathrm{ft})\end{array}$ & $\begin{array}{c}<2438.22 \mathrm{~m}_{\mathrm{b}} \\
(<7999.40 \mathrm{ft})^{b}\end{array}$ & $\begin{array}{r}<2438.22 \mathrm{~m}_{\mathrm{b}} \\
(<7999.40 \mathrm{ft})^{\circ}\end{array}$ & $\begin{array}{l}<2438.22 \mathrm{~m}_{\mathrm{b}} \\
(<7999.40 \mathrm{ft})\end{array}$ & $\begin{array}{l}2438.15 \mathrm{~m} \\
(7999.17 \mathrm{ft}) \\
\end{array}$ & $\begin{array}{l}<2438.22 \mathrm{~m} \\
<<7999.40 \mathrm{ft})\end{array}$ & $\begin{array}{l}<2438.22 \mathrm{~m} \\
(<7999.40 \mathrm{ft})\end{array}$ & \\
\hline RU-08 & $\begin{array}{r}2429.05 \mathrm{~m} \\
(7969.33 \mathrm{ft}) \\
\end{array}$ & $\begin{array}{c}2429.13 \\
(7969.60 \mathrm{ft}) \\
\end{array}$ & $\begin{array}{l}<2429.01 \mathrm{~m} \\
(<7969.18 \mathrm{ft}) \\
\end{array}$ & $\begin{array}{l}<2429.01 \mathrm{~m} \\
(<7969.18 \mathrm{ft}) \\
\end{array}$ & $\begin{array}{l}2428.61 \mathrm{~m} \\
(7967.88 \mathrm{ft})\end{array}$ & $\begin{array}{r}2429.34 \mathrm{~m} \\
(7970.26 \mathrm{ft}) \\
\end{array}$ & $\begin{array}{r}2428.99 \mathrm{~m} \\
(7969.14 \mathrm{ft}) \\
\end{array}$ & \\
\hline
\end{tabular}

Well had less than 1 foot of water, so it was not sampled.

${ }^{b}$ Calculated elevation of total depth of Well RU-07 was incorrect in previous reports and has been corrected. 


\subsection{Quality Control Results}

Field and laboratory QC sample requirements and acceptance criteria are specified in the Rulison QAPP (DOE, 1996c). The laboratory narrative for the third quarter sampling analytical results is included in Appendix B and provides a summary of the results for laboratory QC samples required under the various analytical methods used for the project. The following sections describe the results for field QC samples that are not covered by the laboratory narratives because they are not explicit requirements under the analytical methods used, but they are required for field sampling under the Rulison QAPP (DOE, 1996c).

\subsection{Field Duplicate Samples}

Field duplicate samples are used to monitor the variability associated with sample collection procedures and to provide estimates of the total sampling and analytical precision. A duplicate sample was collected from Well RU-06A during the sampling event. The relative percent differences (RPDs) between analytes detected in the original sample and the same analytes detected in the associated field duplicate sample were calculated and compared against the precision acceptance criteria specified in the Rulison QAPP (DOE, 1996c). The sample and sample duplicate results, calculated RPDs, and precision acceptance criteria are presented in Table 4-1.

Barium was the only analyte detected in the RU-06A sample and the sample duplicate. The Relative Percent Difference for barium was within the precision acceptance criterion of \pm 20 percent specified in the Rulison QAPP (DOE, 1996c). Chromium was not detected in either the sample or the duplicate.

\subsection{Equipment Rinsate Blank Samples}

Equipment rinsate blanks are used to monitor potential cross-contamination associated with inadequate equipment decontamination procedures. At Rulison, new, dedicated, disposable bailers were used at each well, eliminating the possibility of cross-contamination between wells. An equipment rinse blank was prepared using deionized water to rinse a new, dedicated, disposable bailer prior to its use in sampling water from RU-06A. Lead was found in the equipment rinsate blank at $3.5 \mu \mathrm{g} / \mathrm{L}$, and in Wells RU-03, RU-05, an RU-08. Lead has been detected previously in Wells RU-03, RU-05, and RU-08 with no lead in the rinsate sample. Lead was not found in Well RU-06A or its duplicate. Lead has not been detected previously in Well RU-06A. 
Table 4-1

Rulison Site Groundwrater Monitoring Program

Duplicate Sample Comparison:

Third Quarter, 1997

(All results in $\mu \mathrm{g} / \mathrm{L}$ )

\begin{tabular}{|c|c|c|c|c|}
\hline \multirow{2}{*}{ Analyte } & \multicolumn{3}{|c|}{ Well RU-6A } & \multirow{2}{*}{$\begin{array}{c}\text { RPD Acceptance } \\
\text { Criterion }\end{array}$} \\
\hline & $\begin{array}{c}\text { Sample } \\
\text { RUW00114 }\end{array}$ & $\begin{array}{c}\text { Sample Duplicate } \\
\text { RUW00116 }\end{array}$ & RPD $^{1}$ & \\
\hline TPH & $1000 \mathrm{U}$ & $1000 U$ & 0 & \pm 40 \\
\hline Benzene & $1.0 \mathrm{U}$ & 1.00 & 0 & \pm 11 to 24 \\
\hline Toluene & $1.0 \mathrm{U}$ & $1.0 \mathrm{U}$ & 0 & \pm 11 to 24 \\
\hline Ethylbenzene & $1.0 U$ & $1.0 U$ & 0 & \pm 11 to 24 \\
\hline Xylenes & $2.0 \mathrm{U}$ & 2.00 & 0 & \pm 11 to 24 \\
\hline Arsenic & $3.0 \mathrm{U}$ & $3.0 \mathrm{U}$ & 0 & \pm 20 \\
\hline Barium & 114.0 & 114.0 & 0 & \pm 20 \\
\hline Cadmium & $1.0 \mathrm{U}$ & 1.00 & 0 & \pm 20 \\
\hline Chromium & $1.0 \mathrm{U}$ & 1.00 & 0 & \pm 20 \\
\hline Lead & $2.0 \mathrm{U}$ & 2.00 & 0 & \pm 20 \\
\hline Mercury & $0.2 U$ & $0.2 \mathrm{U}$ & 0 & \pm 20 \\
\hline Selenium & $3.0 \mathrm{U}$ & $3.0 \mathrm{U}$ & 0 & \pm 20 \\
\hline Silver & $1.0 \mathrm{U}$ & $1.0 \mathrm{U}$ & 0 & \pm 20 \\
\hline
\end{tabular}

'Relative percent difference

$U=$ Analyte not detected above the specified value

\subsection{Trip Blank Samples}

Trip blanks are used to monitor potential volatile organic compound (VOC) cross-contamination introduced into VOC sample containers through diffusion during sample shipment and storage. Trip blank samples were placed in each container used for shipping BTEX samples. BTEX compounds were not detected in the trip blank from the third quarter sampling event. 


\subsection{Summary and Conclusions}

The analytical data from the third quarter 1997 groundwater sampling event indicate that migration of contaminants from the drilling effluent pond sediments currently does not appear to be occurring. The following is a summary of the third quarter 1997 groundwater sample results:

BTEX Compounds: Benzene and toluene were detected in upgradient Well RU-03. BTEX compounds were not detected in any other third quarter groundwater samples.

Diesel-Range TPH: Diesel-range TPH was not detected in any of the third quarter groundwater samples.

Inorganics: Barium, chromium, and lead were detected in the third quarter 1997 groundwater samples from the upgradient and downgradient wells. Chromium and lead were not detected in downgradient monitoring well (RU-06A). The relatively high levels detected in RU-05 are most likely naturally occurring and the result of the uptake of sediment into the groundwater sample. Since these COPCs were detected in the upgradient well, its presence is not likely to represent migration from the pond sediments. As discussed in Section 3.3, there currently are insufficient data to establish concentration trends or to determine whether barium concentrations in groundwater downgradient from the drilling effluent pond are significantly elevated above background. Statistical trends will be calculated as data are acquired from additional quarterly sampling events. 


\subsection{References}

AEC, see U.S. Atomic Energy Commission.

California State Water Resources Control Board. 1989. Leaking Underground Fuel Tank Field Manual, Guidelines for Site Assessment, Cleanup, and Underground Storage Tank Closure. Sacramento, CA.

DOE, see U.S. Department of Energy.

Eberline, see Eberline Instrument Corporation.

Eberline Instrument Corporation. 1977. Rulison Radiation Contamination Clearance Report. Santa Fe, NM.

EPA, see U.S. Environmental Protection Agency.

ERDA, see U.S. Energy Research and Development Administration.

U.S. Atomic Energy Commission, Nevada Operations Office. 1973. Rulison Site Cleanup Report, NVO-136. Las Vegas, NV.

U.S. Department of Energy. 1996a. Rulison Drilling Effluent Pond Site Long-Term Groundwater Monitoring Plan, DOE/NV-441. Las Vegas, NV: IT Corporation.

U.S. Department of Energy. 1996b. Rulison Site Corrective Action Report, DOE/NV-453. Las Vegas, NV: IT Corporation.

U.S. Department of Energy. 1996c. Rulison Site Quality Assurance Project Plan, Rulison Site, Colorado, DOE/NV-440. Las Vegas, NV: IT Corporation.

U.S. Department of Energy. 1997. Rulison Site Groundwater Monitoring Report, Second Quarter, 1997, DOE/NV-460. Las Vegas, NV: IT Corporation.

U.S. Energy Research and Development Administration, Nevada Operations Office. 1977. Project Rulison Well Plugging and Site Abandonment Final Report, NVO-187.

Las Vegas; NV.

U.S. Environmental Protection Agency. 1983. Methods for Chemical Analysis of Water and Wastes, EPA-600/4-79-020. Cincinnati, OH.

U.S. Environmental Protection Agency. 1990. Test Methods for Evaluating Solid Waste, Physical/Chemical Methods, SW-846, 3rd Edition. Washington, DC: Office of Solid Waste and Emergency Response. 
Appendix A

Purge Water Discharge Permit 
a5-19-1996 17:39 $03 / 19 / 1995$ 16:35
702 es51123 $383-782-0390$

\section{STATE OF COLORADO}

en klomex Convme:

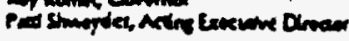

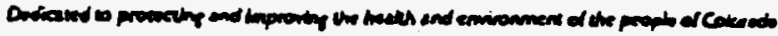

400 Chery Cicuk Or.s.

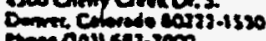

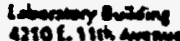

main desisti-secos

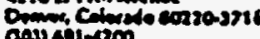

cast coliones

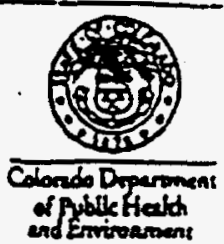

March 19. 1996

Mr. Kavin D. Leay

DOE

Subjec: Reply wo requan for sddition of souree 10 permit COG-31004.

Dear Mr. Leary:

The Divicios the recelved and reviewed your fex of 3/19/95. Since the walte described in your fsi we th

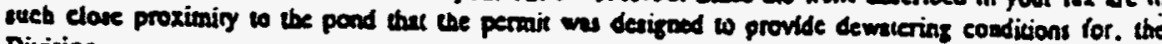
Division

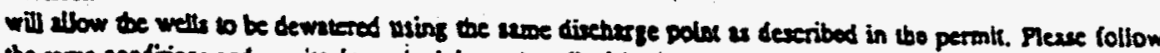

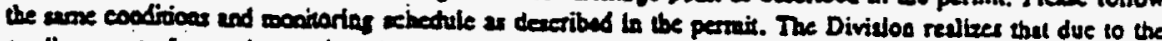

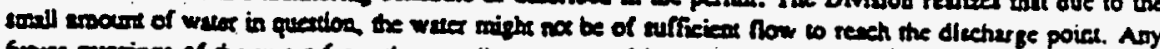
funure purginge of the water from these wolls are cowared by this bewer asd the perwit noted above as long at the permit remeins active and condicion, manibriog achodule and roporing procedure are followed.

Plese feel free to call me \& $(303)+692-3593$ with quatione or comments.

Sincerely.

Toch

Ton Boyee

Environmented Procection Specielia

Permita and Eaforecoeos

WATER QUASTY CONTROL DIVISION

ex.rie 


\section{Appendix B}

Third Quarter 1997 Analytical Results 
Table B-1

Sample Number and Description

\begin{tabular}{|c|l|}
\hline Sample Number & \multicolumn{1}{|c|}{ Sample Location or Description } \\
\hline \hline RUW00114 & Well RU-06A \\
\hline RUW00115 & Trip Blank \\
\hline RUW00116 & Duplicate of RUW00114 at RU-06A \\
\hline RUW00117 & Well RU-03 \\
\hline RUW00118 & Equipment Rinsate \\
\hline RUW00119 & Well RU-05 \\
\hline RUW00120 & Not Collected - Well RU-07 was dry. \\
\hline RUW00121 & Well RU-08 \\
\hline
\end{tabular}




\section{LAS LABORATORIES}

TOTAL PETROLEUM HYDROCARBQNS (TPH)

8015M - TPH

$\begin{array}{ll}\text { Client Sample ID: } & \text { RUWO0114 } \\ \text { Date Collected: } & 21-\text { AUG }-97 \\ \text { Date Analyzed: } & 26-\text { SEP-97 } \\ \text { Date Extracted: } & 28-\text { AUG }-97 \\ \text { Matrix: } & \text { Water }\end{array}$

IAS Sample ID:

Date Received:

Analytical Batch ID: 092597-8015-D-1

Analytical Dilution: 1

Preparation Dilution: 1.0

QC Group :

$8015 \mathrm{M}$ - TPH_52786

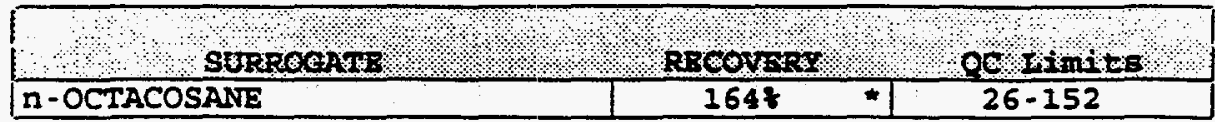

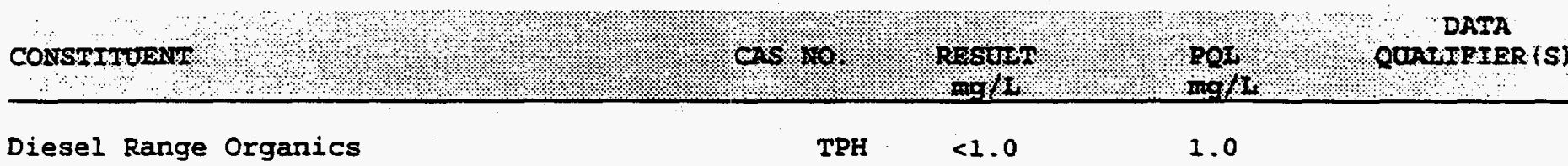

\begin{tabular}{llll}
\hline LJ7781STANDARD & R20106 & Page 1
\end{tabular}

B-2 


\section{LAS LABORATORIES}

TOTAL PETROLEUM HYDROCARBONS (TPH) $8015 M$ - TPH

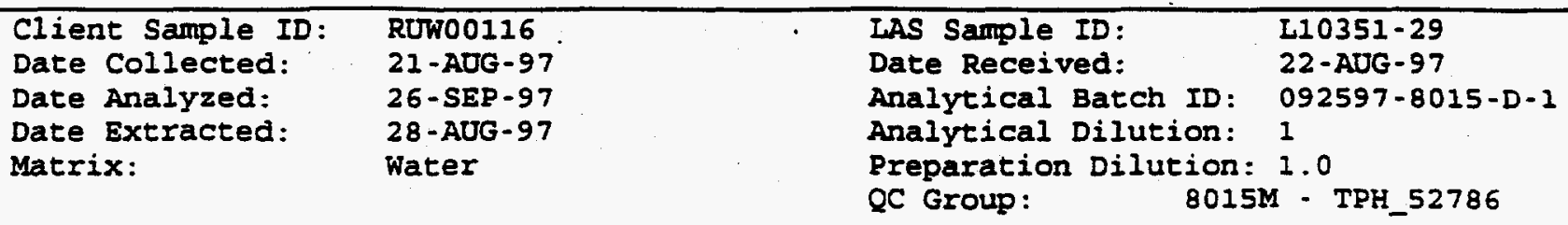

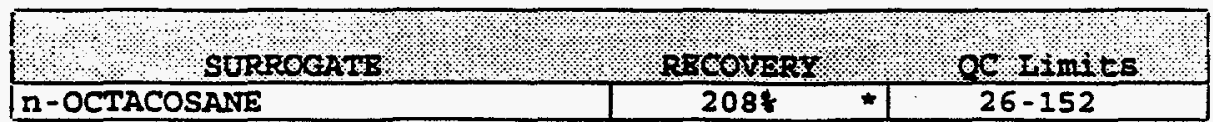

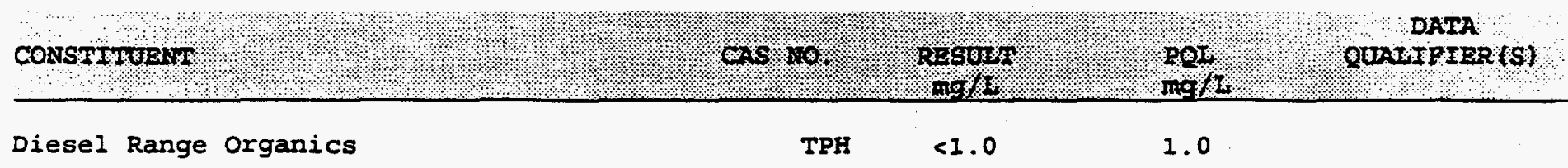

B-3

10/23/97 Revision 7 


\section{LAS LABORATORIES}

TOTAL -PETROLEUM HYDROCARBONS (TPH)

8015M - TPH

\begin{tabular}{|c|c|c|c|c|}
\hline $\begin{array}{l}\text { Client Sample ID: } \\
\text {-Date Collected: } \\
\text { Date Analyzed: } \\
\text { Date Extracted: } \\
\text { Matrix: }\end{array}$ & $\begin{array}{l}\text { RUWOOII7 } \\
21-A O G-97 \\
26-\text { SEP-97 } \\
28 \text {-ADG- } 97 \\
\text { Water }\end{array}$ & $\begin{array}{l}\text { IAS Sample ID: } \\
\text { Date Received: } \\
\text { Analytical Batch } \\
\text { Analytical Dilutic } \\
\text { Preparation Dilut } \\
\text { QC Group: }\end{array}$ & $\begin{array}{l}\text { ID : } \\
\text { on: } \\
\text { ion: } \\
\text { 8015M }\end{array}$ & $\begin{array}{l}\text { L10351-30 } \\
22-A U G-97 \\
092597-8015-D-1 \\
1 \\
1.0 \\
M-\text { TPH } 52786\end{array}$ \\
\hline
\end{tabular}

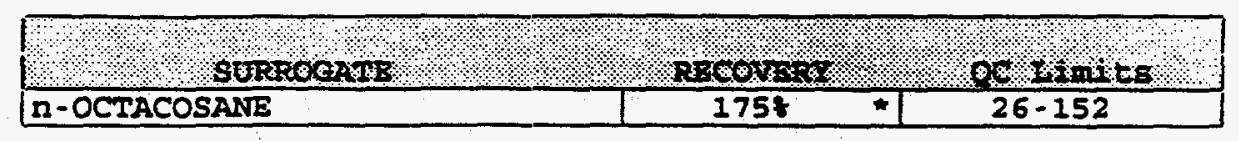

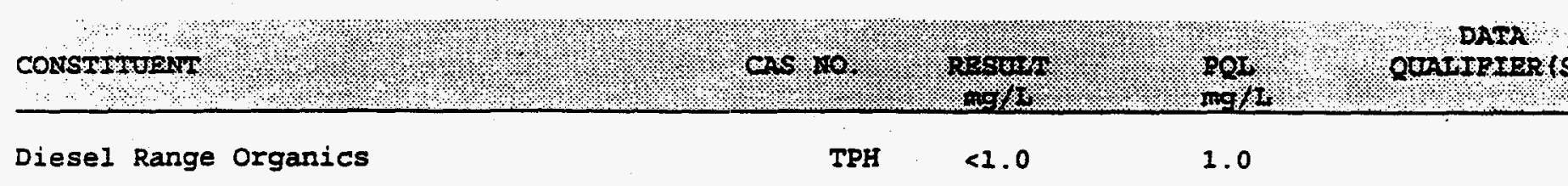

\begin{tabular}{llll}
\hline LJ7781STANDARD & N R20106 & Page 1
\end{tabular}




\section{LAS LABORATORIES}

TOTAL PETROLEUM HYDROCARBỌNS (TPH)

8015M - TPH

\begin{tabular}{|c|c|c|c|}
\hline $\begin{array}{l}\text { Client Sample ID: } \\
\text { Date Collected: } \\
\text { Date Analyzed: } \\
\text { Date Extracted: } \\
\text { Matrix: }\end{array}$ & $\begin{array}{l}\text { RUWOO118 } \\
21 \text {-AUG-97 } \\
26 \text {-SEP-97 } \\
28 \text {-AUG-97 } \\
\text { Water }\end{array}$ & $\begin{array}{l}\text { IAS Sample ID: } \\
\text { Date Received: } \\
\text { Analytical Batch ID: } \\
\text { Analytical Dilution: } \\
\text { Preparation Dilution: } \\
\text { QC Group: }\end{array}$ & $\begin{array}{l}L I 035 I-33 \\
22-A O G-97 \\
092597-8015-D-1 \\
1 \\
1.0 \\
1-T P H \_52786\end{array}$ \\
\hline
\end{tabular}

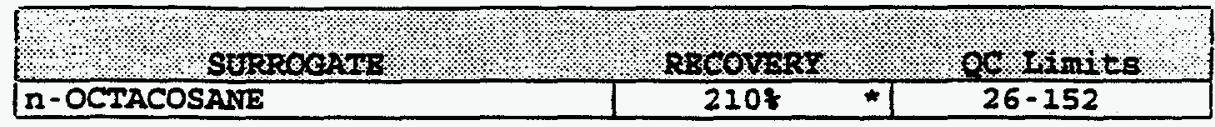

\section{CONSTIIJENT}

$0.0 .10 \%$

rassisto +nos 8

TPÉ

$<1.0$

201

mot 1

DATA

Diesel Range Organics

1.0

1.0 


\section{LAS LABORATORIES}

TOTAL PETROLEUM HYDROCARBONS (TPH)

8015M - TPH

$\begin{array}{llll}\text { Client Sample ID: } & \text { RUWO0119: } & \text { LAS Sample ID: } & \text { L10351-34 } \\ \text { Date Collected: } & 21-A D G-97 & \text { Date Received: } & 22-A O G-97 \\ \text { Date Analyzed: } & 26-\text { SEP-97 } & \text { Analytical Batch ID: 092597-8015-D-1 } \\ \text { Date Extracted: } & 28-A D G-97 & \text { Analytical Dilution: 1 } \\ \text { Matrix: } & \text { Water } & \text { Preparation Dilution: 1. J } \\ & & \text { QC Group: } & \text { 8015M - TPH_52786 }\end{array}$

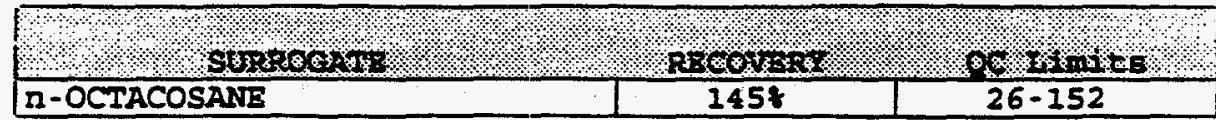

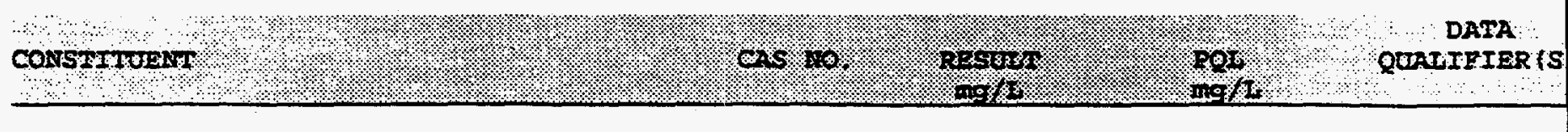

Diesel Range Organics

TPH $\quad<1.0 \quad 1.0$

\begin{tabular}{llll}
\hline LJ7781STANDAFD & R 20106 & Page 1
\end{tabular}

B-6 
TOTAL PETROLLEUM HYDROCARBONS (TPH) 8015M - TPH

$\begin{array}{ll}\text { Client Sample ID: } & \text { RUWO0121 } \\ \text { Date Collected: } & \text { 2I-AOG-97 } \\ \text { Date Analyzed: } & 26-\text { SEP-97 } \\ \text { Date Extracted: } & 28-A 0 G-97 \\ \text { Matrix: } & \text { Water }\end{array}$

LAS Sample ID:

Date Received:

Analytical Batch ID: 092597-8015-D-1

Analytical Dilution: 1

Preparation Dilution: 1.0

QC Group:
L10351-35

2 - AUG - 97

$8015 \mathrm{M}-$ TPH_52786

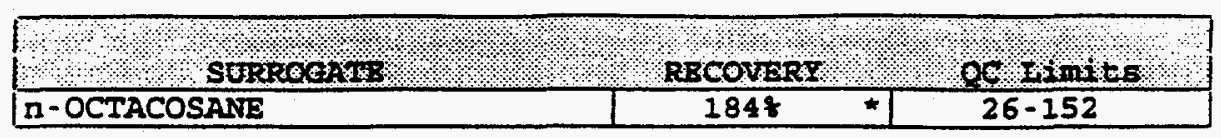

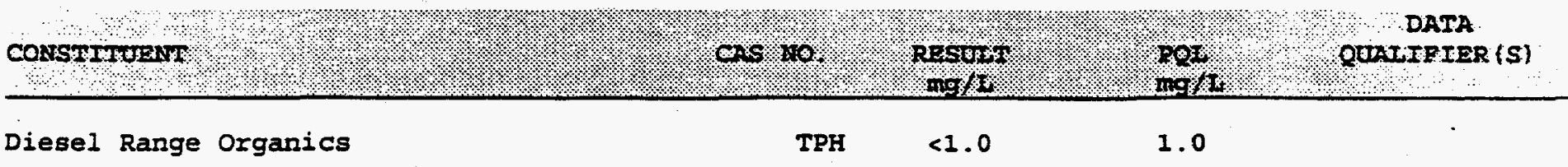

\begin{tabular}{llccc}
\hline LJ7781STANDARD & N & R20106 & Page 1 & $10 / 23 / 97$ Revision 7
\end{tabular}

B-7 


\title{
LAS LABORATORIES
}

\author{
P\&T GAS/BTEX \\ P\&T GAS/BTEX
}

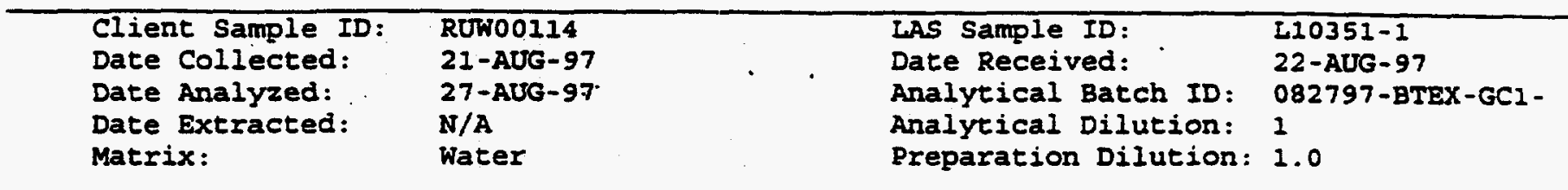

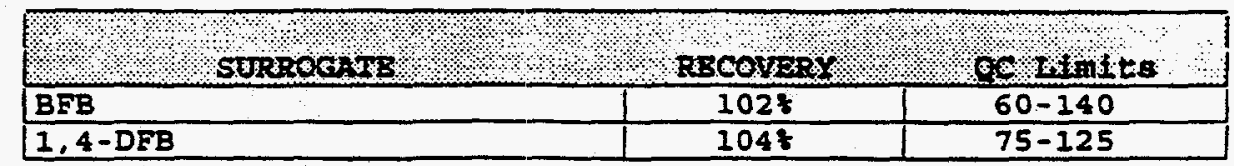

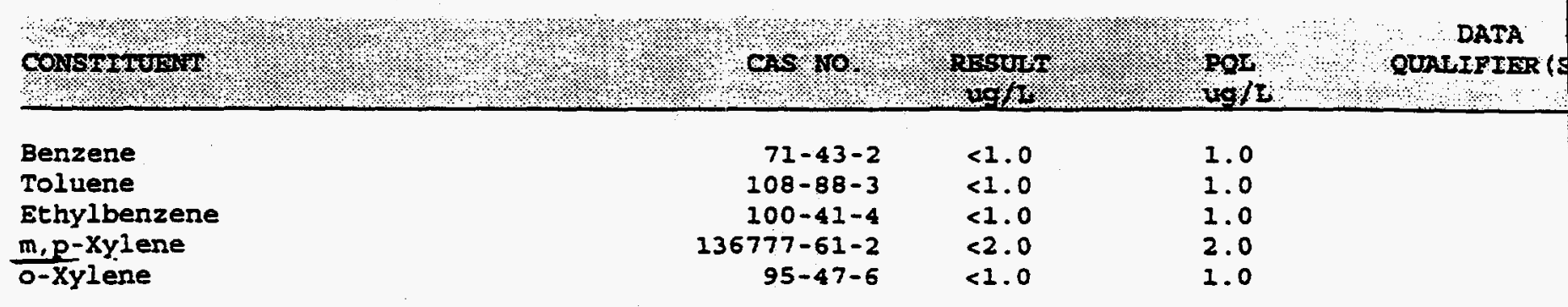




\section{LAS LABORATORIES}

P\&T GAS/BTEX

PET GAS/BTEX

$\begin{array}{llll}\text { Client Sample ID: } & \text { RUW00115 } & \text { LAS Sample ID: } & \text { L10351-4 } \\ \text { Date Collected: } & 21 \text {-AUG-97 } & \text { Date Received: } & 22-\text { AUG-97 } \\ \text { Date Analyzed: } & 28-A U G-97 & \text { Analytical Batch ID: } 082797-B T E X-G C 1- \\ \text { Date Extracted: } & \text { N/A } & \text { Analytical Dilution: } 1 \\ \text { Matrix: } & \text { Water } & \text { Preparation Dilution: } 1.0\end{array}$

\begin{tabular}{|c|c|c|}
\hline ४०८ ४ ८ & & \\
\hline SURROGATE & prooverz: & oc L Limites \\
\hline $\mathrm{BFB}$ & 984 & $60-140$ \\
\hline $1,4-D F B$ & 1048 & $75-125$ \\
\hline
\end{tabular}

\begin{tabular}{|c|c|c|c|c|}
\hline 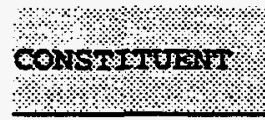 & $1.4 \%$ & 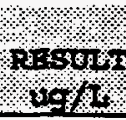 & $\begin{array}{l}\mathrm{POH} \\
\mathrm{x} / \mathrm{g} / \mathrm{s}\end{array}$ & QUAIIFIER (S) \\
\hline Benzene & $71-43-?$ & $<1.0$ & 1.0 & \\
\hline Toluene & $208-88-3$ & $<1.0$ & 1.0 & \\
\hline Ethylbenzene & $100-41=$ & $<1.0$ & 1.0 & \\
\hline $\mathrm{m}, \mathrm{p}$-xylene & $136777-61-2$ & $<2.0$ & 2.0 & \\
\hline o-Xylene & $95-47-6$ & $<1.0$ & 1.0 & \\
\hline
\end{tabular}

\begin{tabular}{llll}
\hline LJ7707BTEX & N & R19778 & Page 1
\end{tabular}

B-9

10/23/97 Revision 7 
P\&T GAS/BTEX

PET GAS/BTEX

\begin{tabular}{|c|c|c|c|}
\hline $\begin{array}{l}\text { Client Sample ID: } \\
\text { Date Collected: } \\
\text { Date Analyzed: } \\
\text { Date Extracted: } \\
\text { Matrix: }\end{array}$ & $\begin{array}{l}\text { RUW00116 } \\
21 \text {-AUG-97 } \\
28 \text {-AUG-97 } \\
\text { N/A } \\
\text { Water }\end{array}$ & $\begin{array}{l}\text { LAS Sample ID: } \\
\text { Date Received: } \\
\text { Analytical Batch ID: } \\
\text { Analytical Dilution: } \\
\text { Preparation Dilution: }\end{array}$ & $\begin{array}{l}\text { L10351-7 } \\
22-A U G-97 \\
082797-B T E X-G C 1- \\
1 \\
1.0\end{array}$ \\
\hline
\end{tabular}

\begin{tabular}{|c|c|c|}
\hline ४। SUTRROGATR8 & RECOUERY & OC Limito \\
\hline BFB & 958 & $60-140$ \\
\hline $1,4-\mathrm{DFB}$ & 1028 & $75-125$ \\
\hline
\end{tabular}

Constrpyrs?
Benzene
Toluene
Ethylbenzene
m, -Xylene
o-Xylene

CAS 110 . 1 , RESELE?

PQL. ug $/ 2$.

DATA

o-Xylene

$$
\begin{array}{r}
71-43-2 \\
108-88-3 \\
100-41-4 \\
136777-61-2 \\
95-47-6
\end{array}
$$

$<1.0$

ug/t:

OUALITIER (S

$<1.0$

$<1.0$

$<2.0$

$<1.0$

1.0

1.0

1.0

2.0

1.0 


\section{LAS LABORATORIES}

P\&T GAS/BTEX

PET GAS/BTEX

$\begin{array}{llll}\text { Client Sample ID: } & \text { RUWO0117 } & \text { LAS Sample ID: } & \text { L10351-10 } \\ \text { Date Collected: } & 21 \text {-AUG-97 } & \text { Date Received: } & 22-A U G-97 \\ \text { Date Analyzed: } & 28-A U G-97 & \text { Analytical Batch ID: } 082797-B T E X-G C 1- \\ \text { Date Extracted: } & \text { N/A } & \text { Analytical Dilution: } 1 \\ \text { Matrix: } & \text { Water } & \text { Preparation Dilution: } 1.0\end{array}$

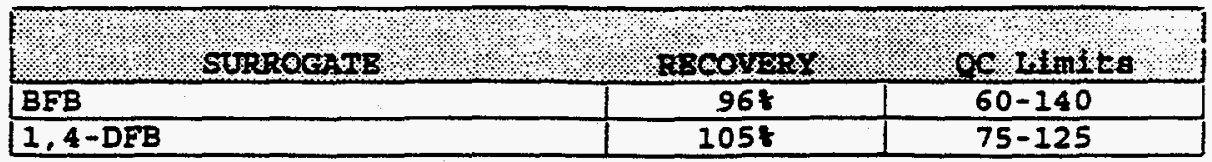

\begin{tabular}{|c|c|c|c|}
\hline CORSFETUBATI & ers 100. & Ressorst. & uon \\
\hline Benzene & $71-43-2$ & 2.5 & 1.0 \\
\hline Toluene & $108-88-3$ & 3.9 & 1.0 \\
\hline Ethylbenzene & $100-41-4$ & $<1.0$ & 1.0 \\
\hline m,p-xylene & $136777-61-2$ & $<2.0$ & 2.0 \\
\hline o-Xylene & $95-47-6$ & $<1.0$ & 1.0 \\
\hline
\end{tabular}

\section{Page 1}

B-11
DATA

QUAIIETERR (S) $0,1 \mathrm{~s}$
1.0
2.0
1.0 


\section{LAS LABORATORIES}

P\&T GAS/BTEX

PET GAS/BTEX

$\begin{array}{llll}\text { Client Sample ID: } & \text { RUW00118 } & \text { LAS Sample ID: } & \text { L10351-19 } \\ \text { Date Collected: } & 21-A U G-97 & \text { Date Received: } & 22-A U G-97 \\ \text { Date Analyzed: } & 28-A U G-97 & \text { N/A } & \text { Analytical Batch ID: } 082797-B \text { TEX-GC1- } \\ \text { Date Extracted: } & \text { Water } & \text { Analytical Dilution: } & 1 \\ \text { Matrix: } & \text { Preparation Dilution: I.0 }\end{array}$

\begin{tabular}{|c|c|c|}
\hline मे. & Fiscosis & 00, f int $t \mathrm{ts}$ \\
\hline BFB & 947 & $60-140$ \\
\hline $1,4-\mathrm{DFB}$ & $102 \%$ & $75-125$ \\
\hline
\end{tabular}

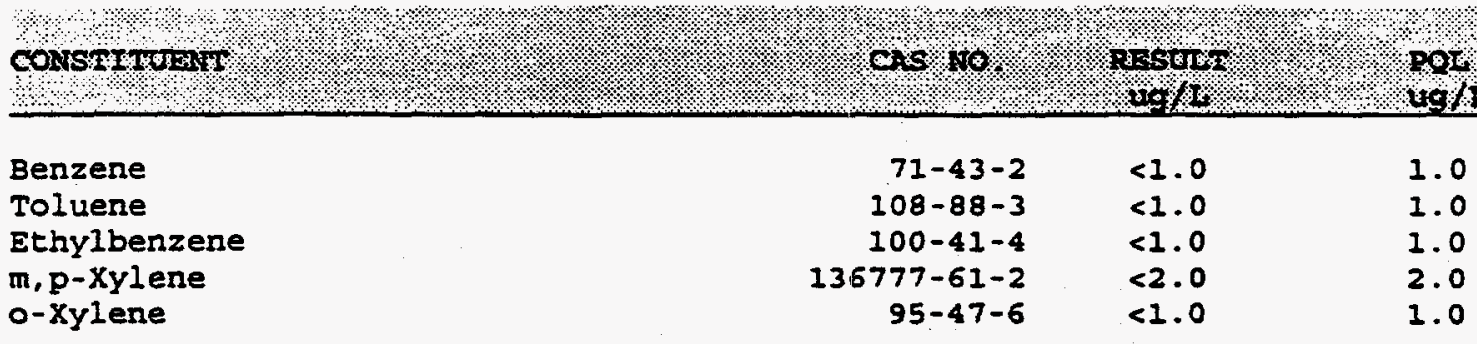


P\&T GAS/BTEX

P\&T GAS/BTEX

$\begin{array}{llll}\text { Client Sample ID: } & \text { RUWO0119 } & \text { LAS Sample ID: } & \text { L10351-22 } \\ \text { Date Collected: } & 21-A U G-97 & \text { Date Received: } & 22-\text { AUG-97 } \\ \text { Date Analyzed: } & 28-A U G-97 & \text { Analytical Batch ID: 082797-BTEX-GC1- } \\ \text { Date Extracted: } & \text { N/A } & \text { Analytical Dilution: } 1 \\ \text { Matrix: } & \text { Water } & \text { Preparation Dilution: } 1.0\end{array}$

\begin{tabular}{|c|c|c|}
\hline "ै. & PL & $0,8 \% \sin \alpha t \theta$ \\
\hline BFB & 994 & $60-140$ \\
\hline $1,4-D F B$ & $108 \%$ & $75-125$ \\
\hline
\end{tabular}

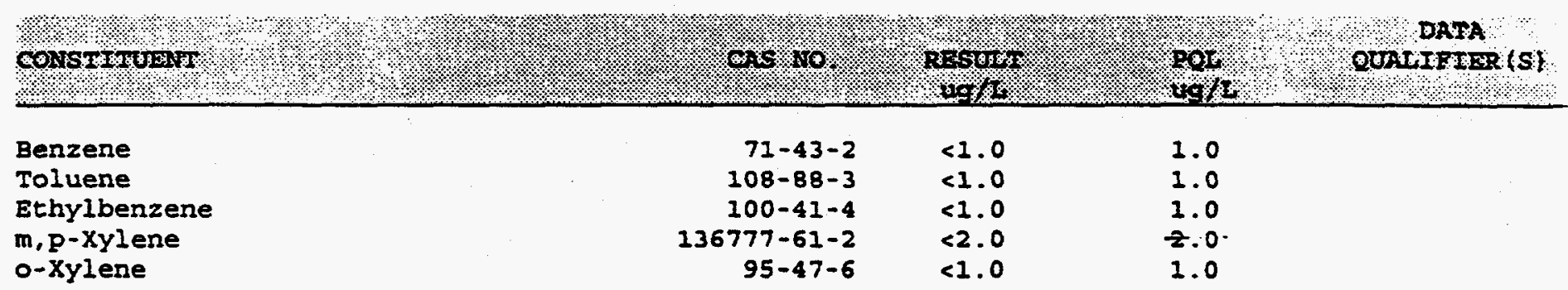

Page 1

B-13
10/23/97 Revision 7 


\section{LAS LABORATORIES}

P\&T GAS/BTEX

PET GAS/BTEX

\begin{tabular}{|c|c|c|c|}
\hline $\begin{array}{l}\text { Client Sample ID: } \\
\text { Date Collected: } \\
\text { Date Analyzed: } \\
\text { Date Extracted: } \\
\text { Matrix: }\end{array}$ & $\begin{array}{l}\text { RUHOO121 } \\
21-A U G-97 \\
28-A U G-97 \\
\text { N/A } \\
\text { Water }\end{array}$ & $\begin{array}{l}\text { LAS Sample ID: } \\
\text { Date Received: } \\
\text { Analytical Batch ID: } \\
\text { Analytical Dilucion: } \\
\text { Preparation Dilution: }\end{array}$ & $\begin{array}{l}210351-25 \\
22-A U G-97 \\
082797-B T E X-G C 1- \\
1 \\
1.0\end{array}$ \\
\hline
\end{tabular}

\begin{tabular}{|c|c|c|}
\hline 1. & 1300vere & 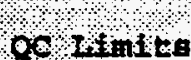 \\
\hline BEB & 978 & $60-140$ \\
\hline $1,4-D F B$ & $103 \%$ & $75-125$ \\
\hline
\end{tabular}

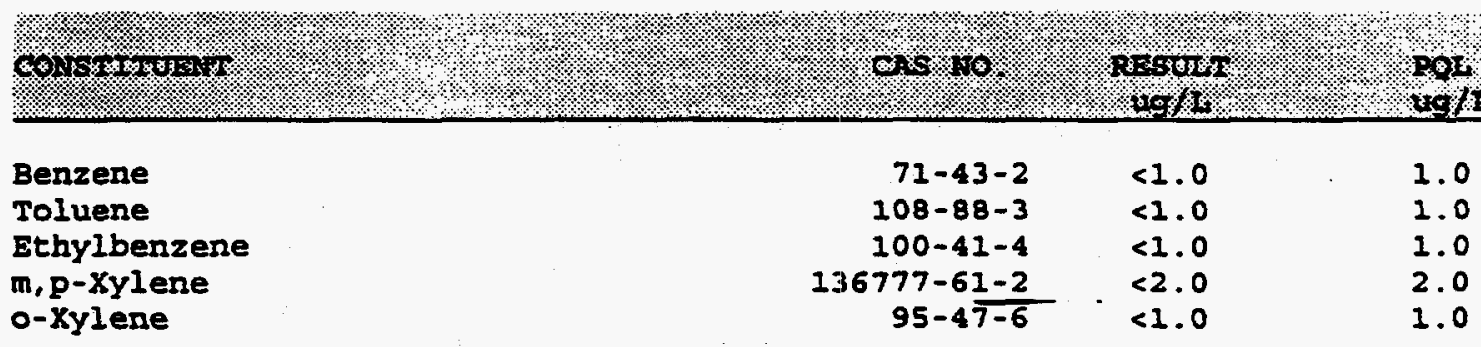




\section{CLP}

1

INORGANIC ANALYSES DATA SHEET
Lab Name: L.A.S

Contract: IT_INTERNA

Lab Code: LOCK

Case No.: 822IT

Matrix (soil/waterl-s

WATER

Level (low/med):

LOW

0.0

\% Solids : SAS NO.:
CLIENT ID NO.

RUWO0114

SDG No.: L10351

Lab Sample ID: L10351-36

Date Received: 08/22/97

Concentration Units (ug/L or $\mathrm{mg} / \mathrm{kg}$ dry weight): UG/L_

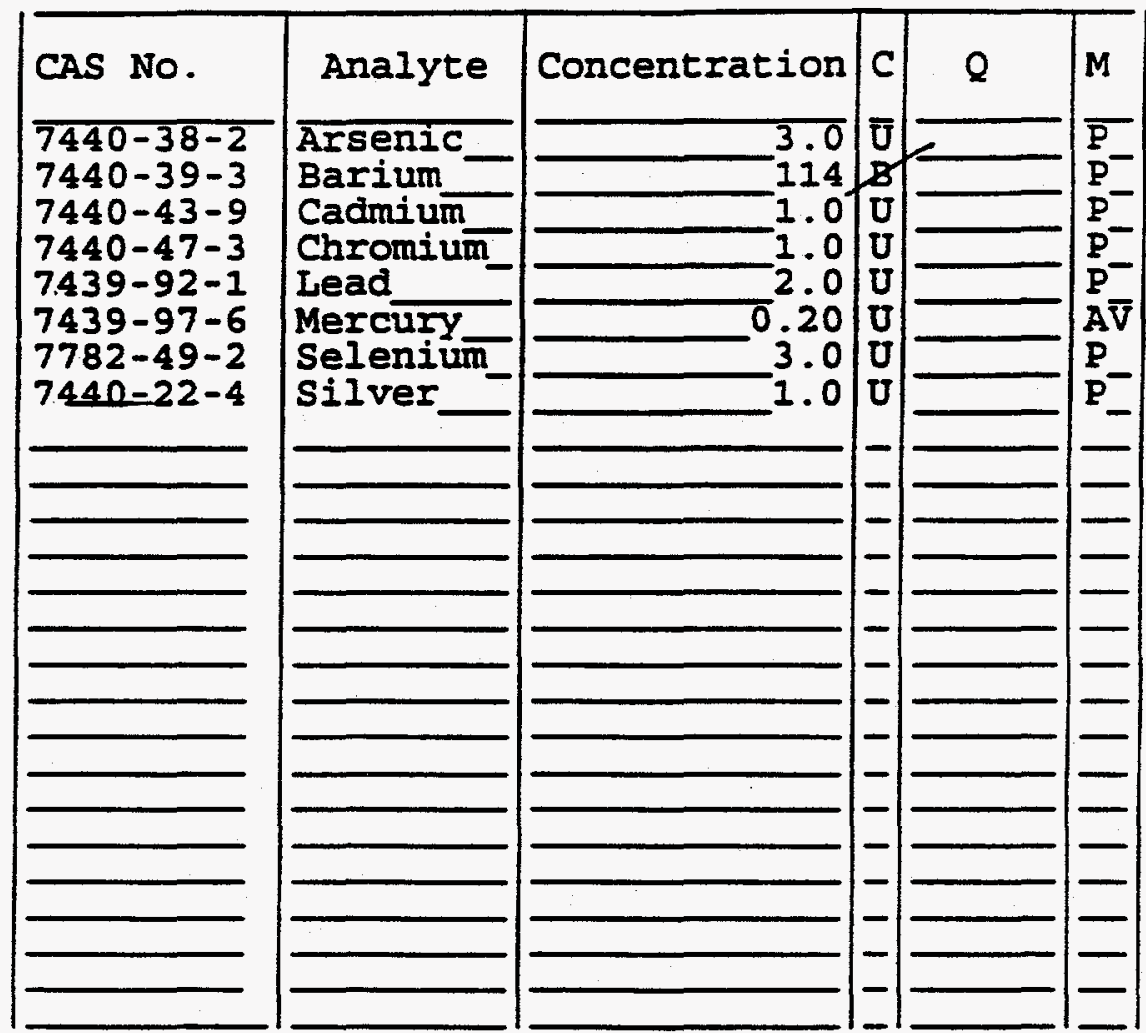

Color Before: COLORLESS

Clarity Before: CLEAR

Texture:

Color After: YELLOW

Clarity After: CLEAR_

Artifacts : Comments :

FORM I - IN

B-15

10/23/97 Revision 7

$\operatorname{nก} 24$ 
1 INORGANIC ANALYSES DATA SHEET
CLIENT ID NO.

\section{RUW00116}

Contract: IT_INTERNA

SDG NO.: L10351
Matrix (soil/water):- WATER

Level (low/med): LOW

Solids : $-0.0$
Case No.: 822IT_ SAS No.:
Lab Sample ID: L10351-37

Date Received: 08/22/97

Concentration Units (ug/L or $\mathrm{mg} / \mathrm{kg}$ dry weight): UG/L_

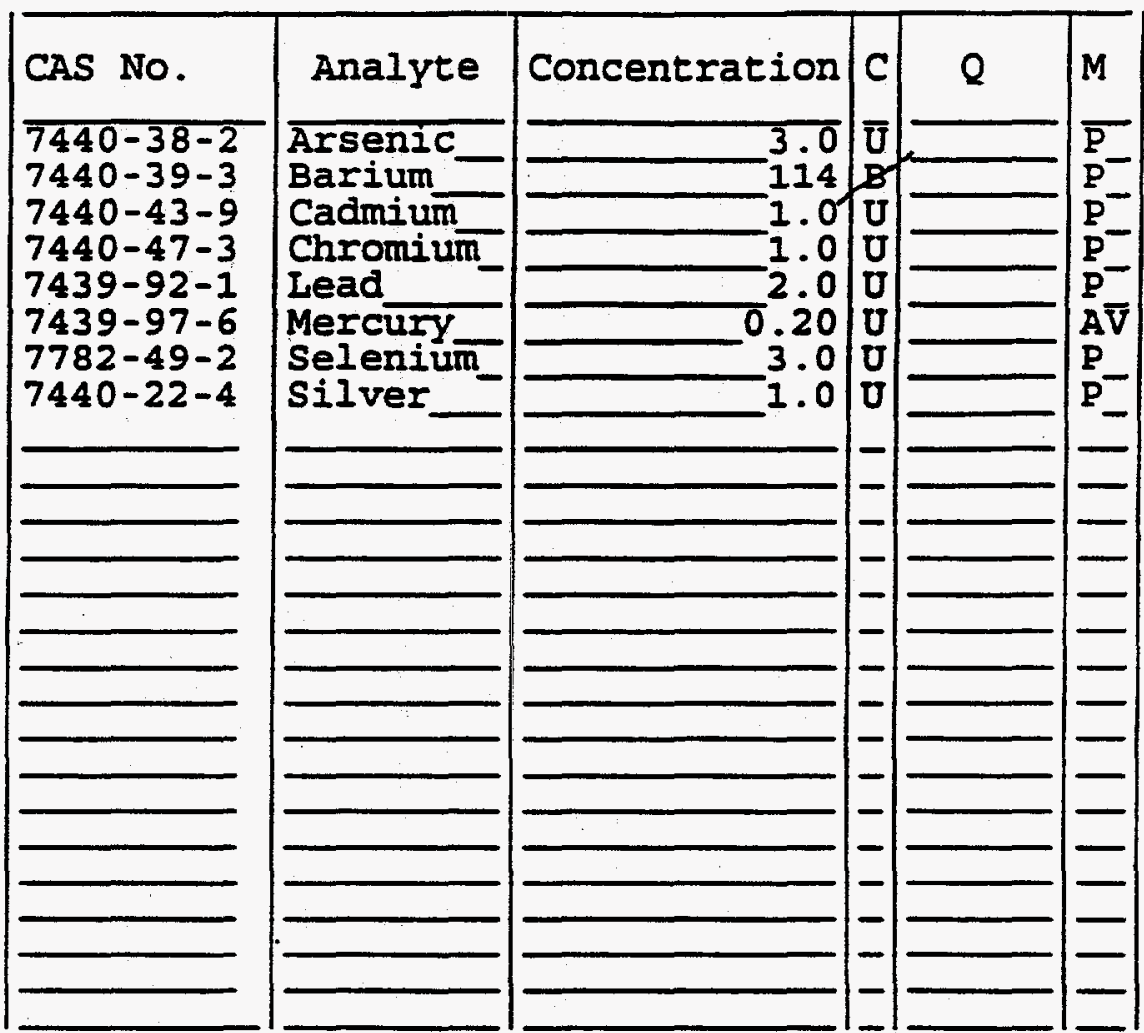

Color Before: COLORLESS

Clarity Before: CLEAR

Texture:

Color After: YELLOW

Clarity After: CLEAR_

Artifacts :

Comments :

FORM I - IN

10/23/97 Revision 7

B-16

$0 \div 35$ 


\section{CLP \\ 1 \\ INORGANIC ANALYSES DATA SHEET}

CLIENT ID NO.

RUW00117
Lab Name: 'L.A.S Contract: IT_INTERNA

Lab Code: LOCK Case No.: 822IT_

Matrix (soil/water):- HATER

Level (low/med) : LOW

: Solids :
0.0
SAS NO.:

Lab Sample ID: L10351-38

Date Received: $08 / 22 / 97$

Concentration Units (ug/L or $\mathrm{mg} / \mathrm{kg}$ dry weight) : UG/L_

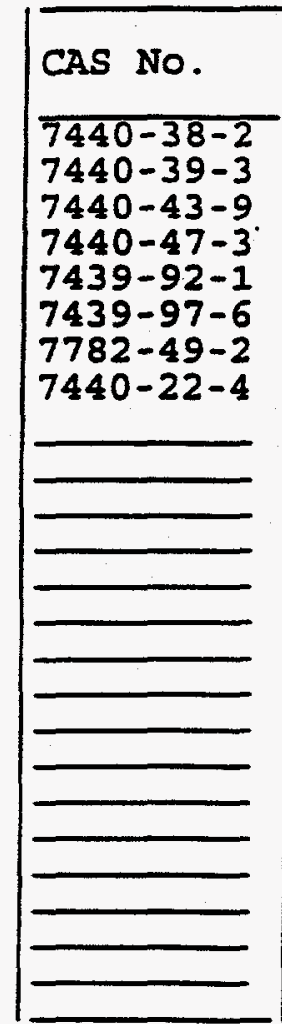

Color Before: BROWN

Color After: YELLOW

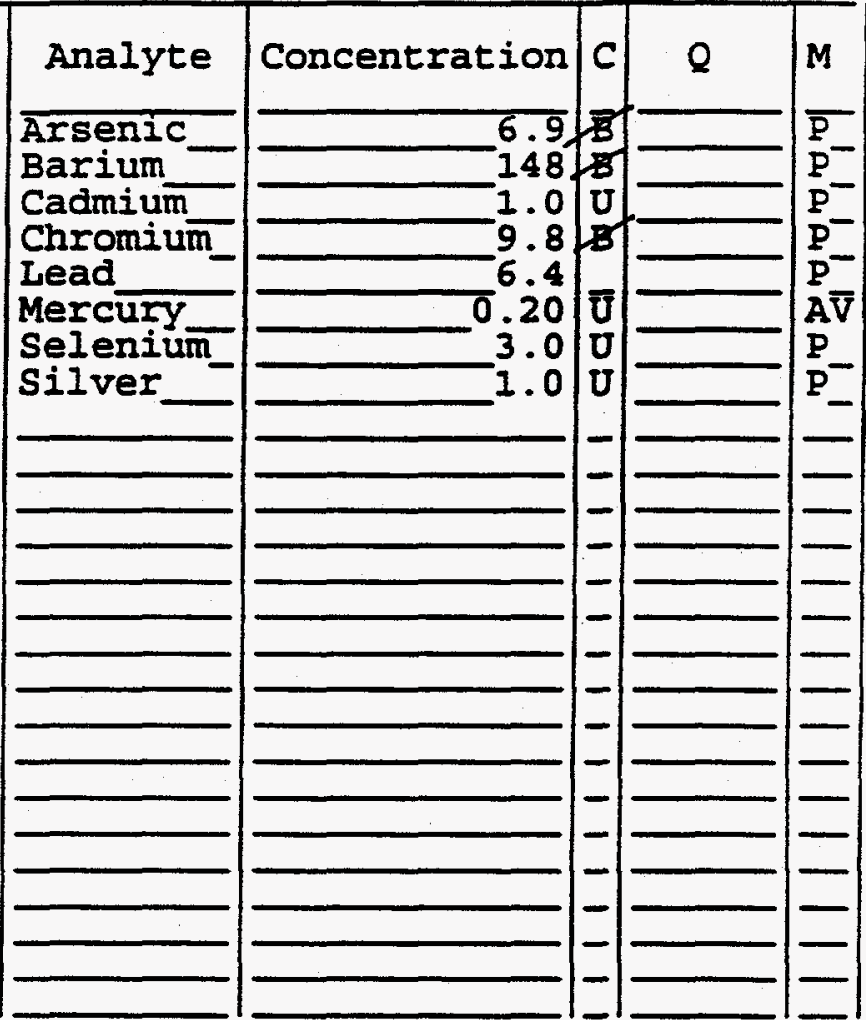

Clarity Before: CLOUDY

Texture:

Clarity After: CLEAR

Artifacts :

Comments :

FORM I - IN 
CLP
$\stackrel{1}{1}$ INORGANIC ANALYSES DATA SHEET

Lab Name: L.A.S Contract: IT_INTERNA Case No.: 82.2IT_ SAS No.: Lab Sample ID: L10351-4I Date Received: 08/22/97
SDG No.: L10351
RUW00118

CLIENT ID NO.

Concentration Units (ug/L or $\mathrm{mg} / \mathrm{kg}$ dry weight) : UG/L_

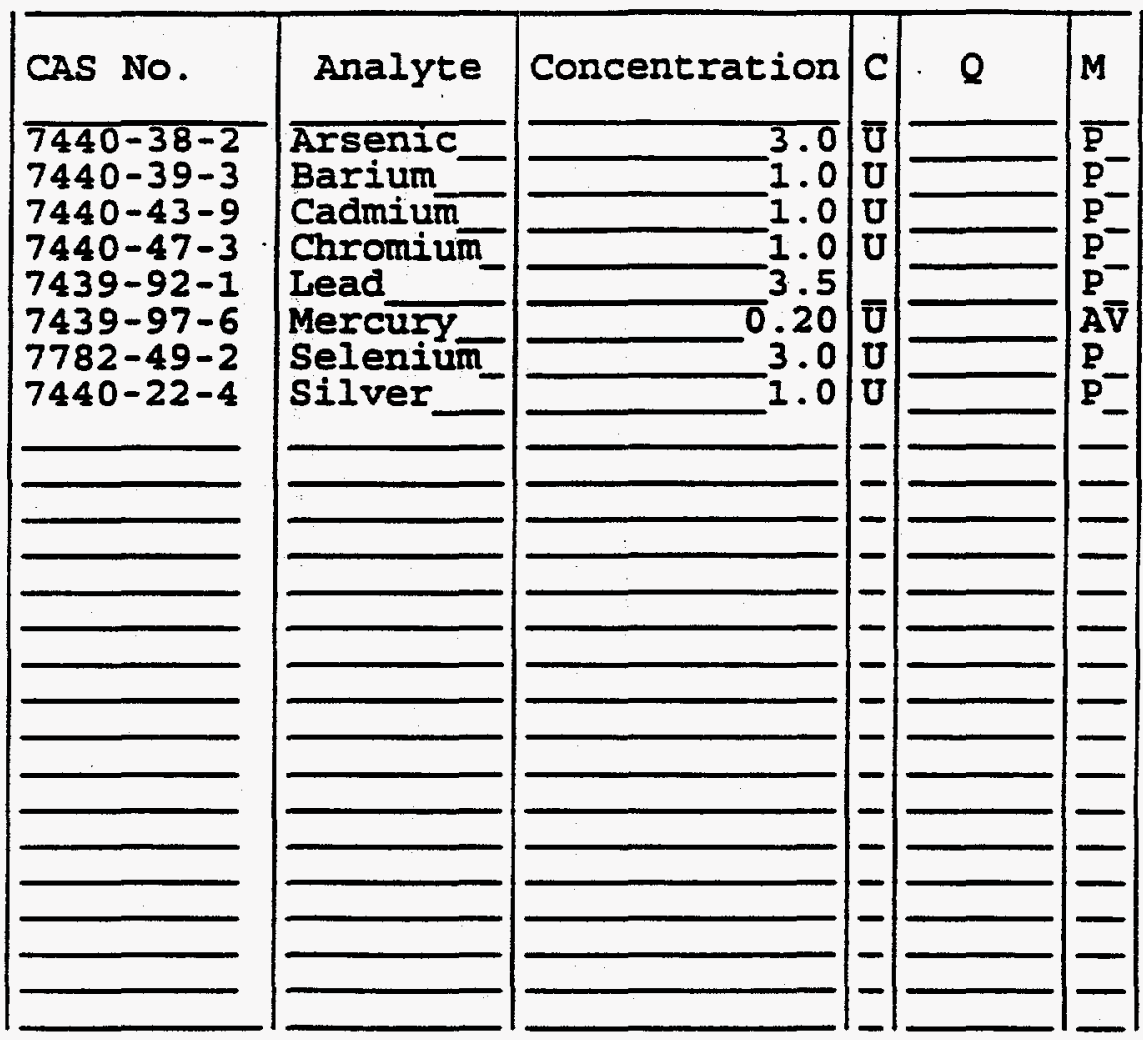

Color Before: COLORLESS

Color After: YELLOW
Clarity Before: CIEAR Clarity After: CLEAR_ Matrix (soil/water): WATER Level (low/med) : LOW

\% Solids :

$-0.0$

Lab Code: LOCK

Comments :

FORM I - IN

10/23/97 Revision 7 
Lab Name: L.A.S

Contract: IT_INTERNA

RUW00119

Lab Code: LOCK Case No.: 822IT_ SAS No.:

CLIENT ID NO.

Matrix (soil/water): WATER

Level (low/med) : LOW

\% Solids :
Lab Sample ID: II0351-42_

Date Received: 08/22/97

Concentration Units (ug/L or $\mathrm{mg} / \mathrm{kg}$ dry weight): UG/L_

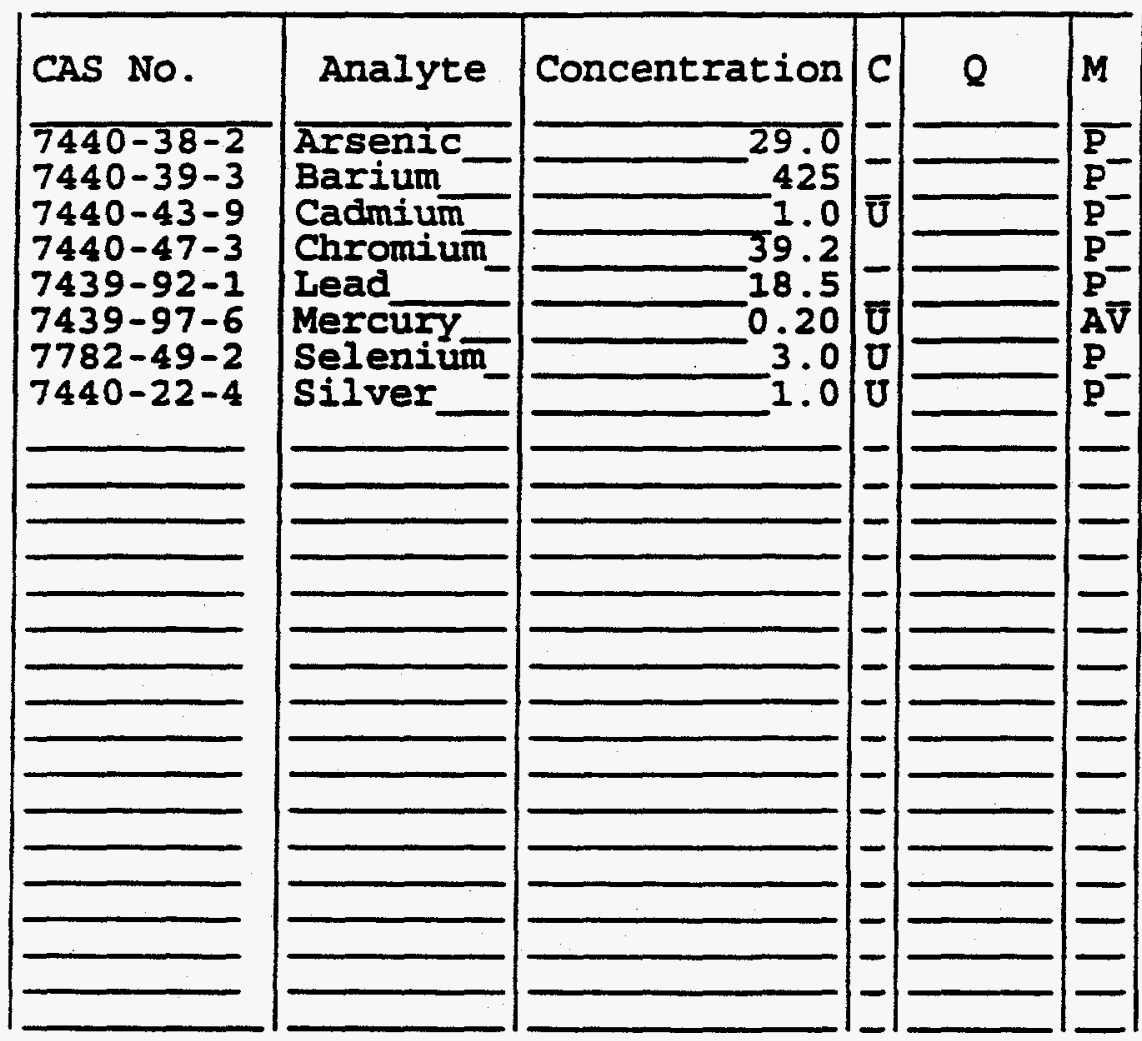

Color Before: BROWN

Color After: YELLOW
Clarity Before: CLOUDY

Clarity After: CLEAR_
Texture :

Artifacts :

Comments :

FORM I - IN

10/23/97 Revision 7

B-19

0028 


\section{CLP}

Lab Name: L.A.S

Contract: IT_INIERNA

Lab Code: LOCK Case No.: 822IT_ SAS NO.:

Matrix (soil/water).: WATER

Level (low/med): LOW

\% Solids :
RUWO0121

SDG No.: L10351

CLIENT ID NO.

Lab Sample ID: L10351-43

Date Received: 08/22/97

Concentration Units (ug/L or $\mathrm{mg} / \mathrm{kg}$ dry weight): UG/L_

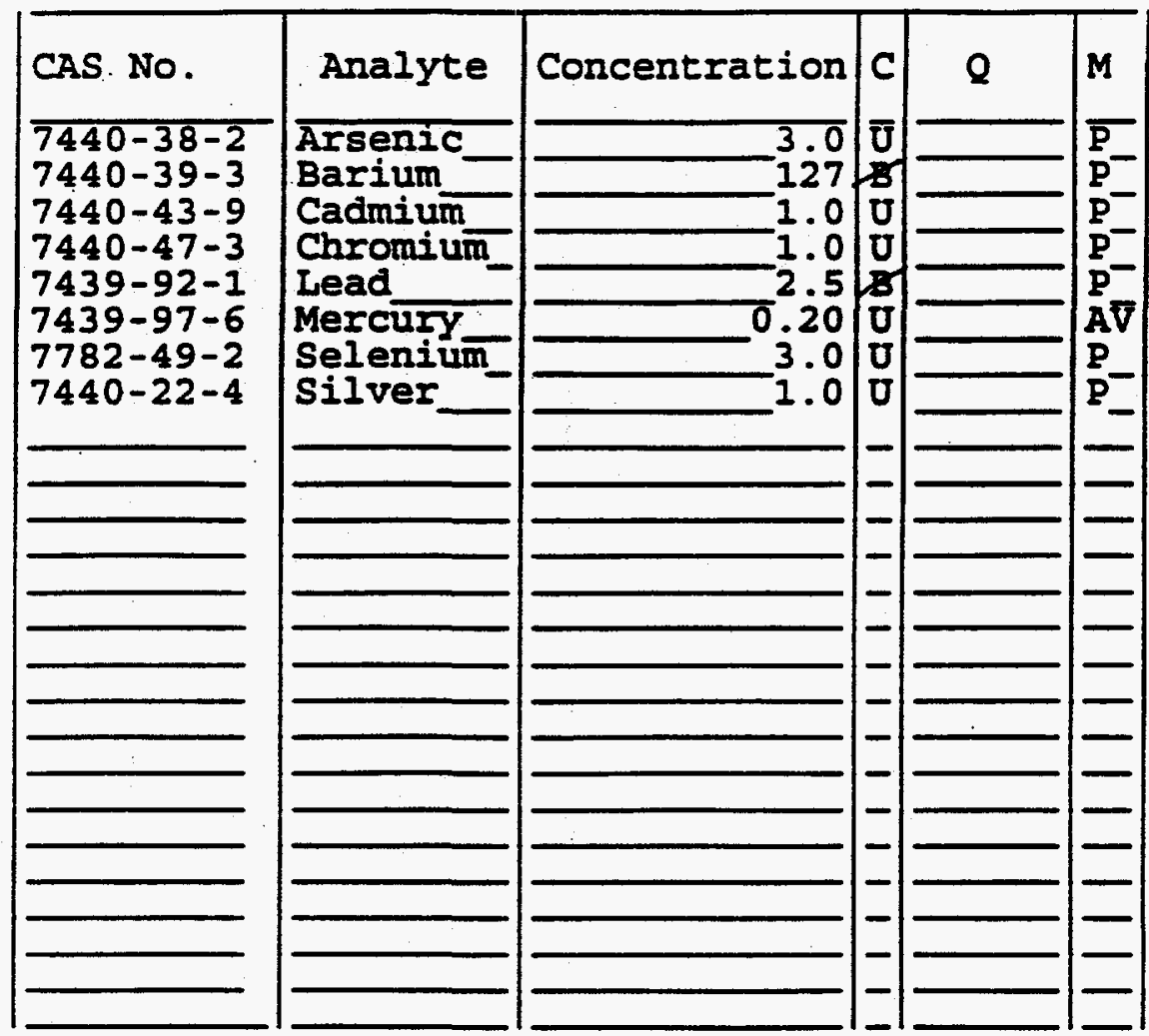

Color Before: COLORLESS

Color After: YELLOW
Clarity Before: CLEAR

Clarity After: CLEAR
Texture:

Artifacts :

Comments :

FORM I - IN 
Distribution List

Copies

DOE/Nevada Operations Office

Technical Information Resource Center

P.O. Box 98518

Las Vegas, Nevada 89193-8518

U.S. Department of Energy

Office of Scientific and Technical Information

175 Oak Ridge Turnpike

Post Office Box 62

Oak Ridge, Tennessee 37831

Glenn Mallory

Colorado Department of Public Health \& Environment 4300 Cherry Creek Drive, S.

Denver, Colorado 80222-1530

Donna Stoner

Industrial Hygienist

Solid Waste Section

Hazardous Materials and Waste Management Division 722 South $6^{\text {th }}$ Street, Room 232

Grand Junction, Colorado 81501

Ann-Marie Welcher

IT Corporation

2621 Losee Rd., Bldg. B-1, Ste. 3050-01

N. Las Vegas, NV 89030

Rick Deshler

IT Corporation

2621 Losee Rd., Bldg. B-1, Ste. 3050-01

N. Las Vegas, NV 89030 